\title{
Fano-Kondo resonance versus Kondo plateau in an Aharonov-Bohm ring with an embedded quantum dot
}

\author{
Mikio Eto \\ Faculty of Science and Technology, Keio University, 3-14-1 Hiyoshi, Kohoku-ku, Yokohama 223-8522, Japan \\ Rui Sakano \\ Institute for Solid State Physics, University of Tokyo, Kashiwa, Chiba 277-8581, Japan
}

(Received 10 September 2020; revised 4 November 2020; accepted 5 November 2020; published 1 December 2020)

\begin{abstract}
We theoretically examine the transport through an Aharonov-Bohm ring with an embedded quantum dot (QD), the so-called QD interferometer, to address two controversial issues regarding the shape of the Coulomb peaks and measurement of the transmission phase shift through a QD. We extend a previous model [B. R. Bulka and P. Stefański, Phys. Rev. Lett. 86, 5128 (2001); W. Hofstetter, J. König, and H. Schoeller, ibid. 87, 156803 (2001)] to consider multiple conduction channels in two external leads, $L$ and $R$. We introduce a parameter $p_{\alpha}\left(\left|p_{\alpha}\right| \leqslant 1\right)$ to characterize a connection between the two arms of the ring through lead $\alpha(=L, R)$, which is the overlap integral between the conduction modes coupled to the two arms. First, we study the shape of a conductance peak as a function of energy level in the $\mathrm{QD}$, in the absence of electron-electron interaction $U$. We show an asymmetric Fano resonance for $\left|p_{L, R}\right|=1$ in the case of single conduction channel in the leads and an almost symmetric Breit-Wigner resonance for $\left|p_{L, R}\right|<0.5$ in the case of multiple channels. Second, the Kondo effect is taken into account by the Bethe ansatz exact solution in the presence of $U$. We precisely evaluate the conductance at temperature $T=0$ and show a crossover from an asymmetric Fano-Kondo resonance to the Kondo plateau with changing $p_{L, R}$. Our model is also applicable to the multiterminal geometry of the QD interferometer. We discuss the measurement of the transmission phase shift through the QD in a three-terminal geometry by a "double-slit experiment." We derive an analytical expression for the relation between the measured value and the intrinsic value of the phase shift.
\end{abstract}

DOI: 10.1103/PhysRevB.102.245402

\section{INTRODUCTION}

In the mesoscopic physics, an Aharonov-Bohm (AB) ring with an embedded quantum dot (QD), the so-called QD interferometer, has been intensively studied to elucidate the coherent transport through a QD with discrete energy levels and strong Coulomb interaction [1-4]. Controversial issues still remain regarding the transport through the interferometer despite long-term experimental and theoretical studies. We theoretically revisit these issues by generalizing a previous model to consider multiple conduction channels in external leads and a multiterminal geometry.

We first discuss the shape of Coulomb peaks, i.e., conductance $G$ as a function of gate voltage attached to the QD to control the energy levels electrostatically. Kobayashi et al. observed an asymmetric shape of the Coulomb peaks, which has a peak and dip in accordance with the Fano resonance, using a QD interferometer [5]. The Fano resonance stems from the interference between a discrete energy level in the QD and continuum energy states in the ring [6,7]. Remarkably the resonant shape of the Coulomb peaks changes with a magnetic flux penetrating the ring. However, the other groups observed symmetric Coulomb peaks, which can be fitted to the Lorentzian function of Breit-Wigner resonance [8]. No criteria has been elucidated regarding the Fano or Breit-Wigner resonance in the QD interferometer.

The second issue concerns the measurement of the transmission phase shift through a QD using the QD interferometer as a double-slit experiment. It is well known that the phase shift cannot be observed by the interferometer in the twoterminal geometry [1]. This is due to the restriction by the Onsager's reciprocity theorem: Conductance $G$ satisfies $G(\boldsymbol{B})=G(-\boldsymbol{B})$ for magnetic field $\boldsymbol{B}$, or $G(\phi)=G(-\phi)$ for the AB phase $\phi=2 \pi \Phi /(h / e)$ with magnetic flux $\Phi$ penetrating the ring $[3,4]$. The phase measurement was first reported using the interferometer in a four-terminal geometry [2]. In the Kondo regime, the phase shift through the QD should be locked at $\pi / 2$ [9-11]. This phase locking was also investigated experimentally using four- or three-terminal devices [8,12-17]. It is nontrivial, however, how precisely the phase shift is measured using the multiterminal interferometer.

Theoretically, Bulka and Stefański studied Fano and Kondo resonances using a model for the two-terminal QD interferometer, in which a QD is coupled to leads $L$ and $R$ and the leads are directly coupled to each other [18]. Hofstetter et al. found an asymmetric Fano-Kondo resonance by applying the numerical renormalization group calculation to an equivalent model [19]. Their works were followed by many theoretical studies, e.g., to elucidate various aspects of the Kondo effect 

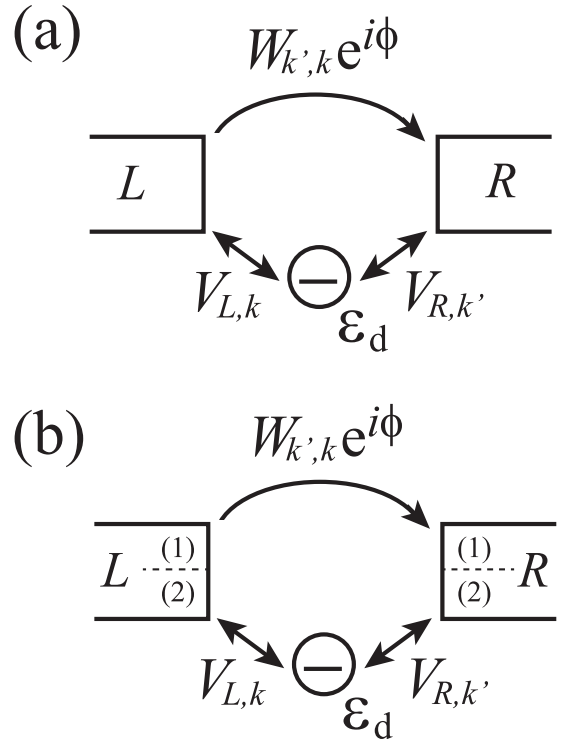

FIG. 1. (a) Model for an $\mathrm{AB}$ ring with an embedded quantum dot (QD), the so-called QD interferometer, in the two-terminal geometry. The lower arm of the ring involves a QD with single energy level $\varepsilon_{d}$, whereas the upper arm directly connects leads $L$ and $R$. The tunnel couplings between the QD and leads, $V_{L, k}, V_{R, k^{\prime}}$ and that through the upper arm $W_{k^{\prime}, k}$, depend on states $k$ in lead $L$ and state $k^{\prime}$ in lead $R$. A magnetic flux $\Phi$ penetrating the ring is taken into account by the AB phase $\phi=2 \pi \Phi /(h / e)$. The electron-electron interaction $U$ works in the QD. (b) Model for the QD interferometer in a fourterminal geometry, with leads $L(1), L(2), R(1)$, and $R(2)$. State $k\left[k^{\prime}\right]$ belongs to lead $L(1)$ or $L(2)$ [R(1) or $R(2)]$. The chemical potentials in the leads are denoted by $\mu_{L}^{(1)}, \mu_{L}^{(2)}, \mu_{R}^{(1)}$, and $\mu_{R}^{(2)}$, respectively, in the formulation in Appendix A. We fix $\mu_{L}^{(1)}=\mu_{L}^{(2)} \equiv \mu_{L}$ and $\mu_{R}^{(1)}=$ $\mu_{R}^{(2)} \equiv \mu_{R}$ with $\mu_{L}-\mu_{R}=e V$ in our calculations.

[20-29], fluctuation theorem [30], and dynamics of electronic states [31]. Recently, the Fano resonance was proposed to detect the Majorana bound states [32,33].

Although the model in Refs. [18,19] was widely used, it is insufficient to describe experimental situations with multiple conduction channels in the leads. In the present paper, we propose an extended model for the QD interferometer to resolve the above-mentioned problems. As shown in Fig. 1(a), our model is the same as the previous model except the tunnel couplings, $V_{L}, V_{R}$, and $W$, depend on the states in leads $L$ and $R$. We show that the state dependence can be disregarded only in the case of single conduction channel in the leads.

Our model yields a parameter $p_{\alpha}\left(\left|p_{\alpha}\right| \leqslant 1\right)$ to characterize a connection between the two arms of the ring through lead $\alpha(=L, R)$, which is the overlap integral between the conduction modes coupled to the upper and lower arms of the ring. First, we examine the shape of a conductance peak in the two-terminal geometry, in the absence of electron-electron interaction $U$ in the QD. We show an asymmetric Fano resonance for $\left|p_{L, R}\right|=1$ in the case of single conduction channel in the leads and an almost symmetric Breit-Wigner resonance at $\left|p_{L, R}\right|<0.5$ in the case of multiple channels. Hence our model could explain the experimental results of both the asymmetric Fano resonance [5] and almost symmetric Breit-Wigner resonance [8], with fitting parameters $p_{L, R}$ to their data.

Second, the transport in the Kondo regime is examined by exploiting the Bethe ansatz exact solution. This method precisely gives us the conductance at temperature $T=0$ in the presence of $U$. We show a crossover from an asymmetric Fano-Kondo resonance [19] to the Kondo plateau with changing $p_{L, R}$.

Our model is also applicable to the multiterminal geometry, where state $k\left[k^{\prime}\right]$ belongs to lead $L(1)$ or $L(2)[R(1)$ or $R(2)]$, as depicted in Fig. 1(b). We discuss the measurement of the transmission phase shift through the QD by a "double-slit experiment" using a three-terminal interferometer. We derive an analytical relation between the observed phase shift and intrinsic phase shift in the absence of $U$. Using a simple model to represent the experiment by Takada et al. $[8,16,17]$, we evaluate the measured phase shift in both the absence and presence of $U$. For $U \neq 0$, we show that the phase locking at $\pi / 2$ is observable in the Kondo regime although it is slightly different from the behavior of the intrinsic phase shift that satisfies the Friedel sum rule.

The organization of the present paper is as follows. In Sec. II, we present our model and calculation method. The parameters $p_{L}$ and $p_{R}$ are introduced, which are relevant to the shape of a conductance peak. We explain the calculation method of the conductance at $T=0$, taking into account the Kondo effect exactly. In Sec. III, the calculated results are given for the shape of the conductance peak in a two-terminal geometry. We discuss the asymmetric Fano resonance versus symmetric Breit-Wigner resonance in the absence of $U$, by changing $p_{L, R}$. We also study the conductance in the Kondo regime in the presence of $U$ and show a crossover from an asymmetric Fano-Kondo resonance to the Kondo plateau. In Sec. IV, we examine the phase measurement in a threeterminal geometry by a double-slit interference experiment. We derive an analytical relation between the measured value and intrinsic value for the transmission phase shift through the QD in the absence of $U$. Two specific models are studied to see a crossover from two- to three-terminal measurement and to simulate the experimental situation using two quantum wires to form the QD interferometer $[8,16,17]$. Section V is devoted to the discussion regarding the justification and generality of our model. The conclusions are given in Sec. VI.

\section{MODEL AND CALCULATION METHOD}

\section{A. Model}

Let us consider a model for the QD interferometer in a twoterminal geometry, depicted in Fig. 1(a). The Hamiltonian is given by

$$
H=H_{\text {dot }}+H_{\text {leads }}+H_{\mathrm{T}}
$$

where

$$
\begin{aligned}
H_{\mathrm{dot}} & =\varepsilon_{d} \sum_{\sigma} n_{\sigma}+U n_{\uparrow} n_{\downarrow}, \\
H_{\text {leads }} & =\sum_{\alpha=L, R} \sum_{k \sigma} \varepsilon_{k} a_{\alpha, k \sigma}^{\dagger} a_{\alpha, k \sigma}
\end{aligned}
$$




$$
\begin{aligned}
H_{\mathrm{T}}= & \sum_{\alpha=L, R} \sum_{k \sigma}\left(V_{\alpha, k} a_{\alpha, k \sigma}^{\dagger} d_{\sigma}+\text { H.c. }\right) \\
& +\sum_{k, k^{\prime}, \sigma}\left(W_{k^{\prime}, k} e^{i \phi} a_{R, k^{\prime} \sigma}^{\dagger} a_{L, k \sigma}+\text { H.c. }\right) .
\end{aligned}
$$

Here $n_{\sigma}=d_{\sigma}^{\dagger} d_{\sigma}$ is the number operator for electrons with spin $\sigma$ in the QD with energy level $\varepsilon_{d}$, where $d_{\sigma}^{\dagger}$ and $d_{\sigma}$ are creation and annihilation operators, respectively. $a_{\alpha, k \sigma}^{\dagger}$ and $a_{\alpha, k \sigma}$ are those for conduction electrons in lead $\alpha(=L, R)$ with state $k$ and spin $\sigma$ whose energy is denoted by $\varepsilon_{k}$. $U$ is the electronelectron interaction in the QD. The tunnel Hamiltonian $H_{\mathrm{T}}$ connects the QD and state $k$ in lead $\alpha$ by $V_{\alpha, k}$ through the lower arm of the ring, whereas it connects state $k$ in lead $L$ and state $k^{\prime}$ in lead $R$ by $W_{k^{\prime}, k}$ through the upper arm of the ring. The AB phase is defined by $\phi=2 \pi \Phi /(h / e)$ for a magnetic flux $\Phi$ penetrating the ring. To make the calculation simple, we decompose $W_{k^{\prime}, k}$ into the contributions from state $k$ in lead $L$ and state $k^{\prime}$ in lead $R$ as

$$
W_{k^{\prime}, k}=\sqrt{w_{R, k^{\prime}} w_{L, k}} .
$$

This separable form is justified for the tight-binding models, as discussed in Sec. V.

For lead $\alpha$, we introduce the following three parameters to describe the contribution to the transport:

$$
\begin{aligned}
\Gamma_{\alpha}(\varepsilon) & =\pi \sum_{k}\left(V_{\alpha, k}\right)^{2} \delta\left(\varepsilon-\varepsilon_{k}\right), \\
x_{\alpha}(\varepsilon) & =\pi \sum_{k} w_{\alpha, k} \delta\left(\varepsilon-\varepsilon_{k}\right), \\
\sqrt{\Gamma_{\alpha}(\varepsilon) x_{\alpha}(\varepsilon)} p_{\alpha}(\varepsilon) & =\pi \sum_{k} V_{\alpha, k} \sqrt{w_{\alpha, k}} \delta\left(\varepsilon-\varepsilon_{k}\right) .
\end{aligned}
$$

We assume that the $\varepsilon$-dependence of these parameters is weak around the Fermi level and simply express $\Gamma_{\alpha}, x_{\alpha}$, and $p_{\alpha}$ for $\varepsilon \approx E_{\mathrm{F}} . \Gamma_{\alpha}\left(x_{\alpha}\right)$ characterizes the strength of tunnel coupling to the QD (coupling through the upper arm of the ring). Using $x=x_{L} x_{R}$, the transmission probability through the upper arm of the ring is given by

$$
T_{\text {upper }}=\frac{4 x}{(1+x)^{2}} .
$$

Concerning $x_{L}$ and $x_{R}$, the physical quantities are always written in terms of $x=x_{L} x_{R}$ in our model [34].

The parameter $p_{\alpha}\left(\left|p_{\alpha}\right| \leqslant 1\right)$ defined by Eq. (8) characterizes a connection between the two arms of the ring through lead $\alpha(=L, R)$. Namely, $p_{\alpha}(\varepsilon)$ is an overlap integral between the conduction mode coupled to the QD and that coupled to the upper arm of the ring in lead $\alpha$ at a given energy $\varepsilon$. The tunnel Hamiltonian $H_{\mathrm{T}}$ in Eq. (4) indicates that these modes are given by $\left|\psi_{\alpha}^{\mathrm{QD}}(\varepsilon)\right\rangle \propto \sum_{k} V_{\alpha, k}|\alpha, k\rangle \delta\left(\varepsilon-\varepsilon_{k}\right)$ and $\left|\psi_{\alpha}^{\text {upper }}(\varepsilon)\right\rangle \propto \sum_{k} \sqrt{w_{\alpha, k}}|\alpha, k\rangle \delta\left(\varepsilon-\varepsilon_{k}\right)$, respectively, where $|\alpha, k\rangle$ is the state $k$ in lead $\alpha$. For $|\psi(\varepsilon)\rangle=\sum_{k} C_{k}|\alpha, k\rangle \delta(\varepsilon-$ $\left.\varepsilon_{k}\right)$ and $|\varphi(\varepsilon)\rangle=\sum_{k} D_{k}|\alpha, k\rangle \delta\left(\varepsilon-\varepsilon_{k}\right)$, we denote the inner product by $\left\langle\psi(\varepsilon) \mid \varphi\left(\varepsilon^{\prime}\right)\right\rangle=\langle\psi \mid \varphi\rangle_{\varepsilon} \delta\left(\varepsilon-\varepsilon^{\prime}\right)$, or $\langle\psi \mid \varphi\rangle_{\varepsilon}=$ $\sum_{k} C_{k}^{*} D_{k} \delta\left(\varepsilon-\varepsilon_{k}\right)$. Then

$$
p_{\alpha}(\varepsilon)=\frac{\left\langle\psi_{\alpha}^{\mathrm{QD}} \mid \psi_{\alpha}^{\text {upper }}\right\rangle_{\varepsilon}}{\sqrt{\left\langle\psi_{\alpha}^{\mathrm{QD}} \mid \psi_{\alpha}^{\mathrm{QD}}\right\rangle_{\varepsilon}\left\langle\psi_{\alpha}^{\text {upper }} \mid \psi_{\alpha}^{\text {upper }}\right\rangle_{\varepsilon}}} .
$$

The interference by the $\mathrm{AB}$ effect is maximal when $\left|p_{L}\right|=$ $\left|p_{R}\right|=1$, whereas it completely disappears when $p_{L}=0$ or $p_{R}=0$. In the previous model [18,19], $\left|\psi_{\alpha}^{\mathrm{QD}}\right\rangle=\left|\psi_{\alpha}^{\text {upper }}\right\rangle$ and thus $p_{\alpha}=1$ since $V_{\alpha, k}$ and $\sqrt{w_{\alpha, k}}$ are constant, irrespective of state $k$. As seen in the following sections, $p_{L}$ and $p_{R}$ play a crucial role in determining the shape of conductance peaks. Although $p_{L}$ and $p_{R}$ should be given by the details of experimental systems, we treat them as parameters as well as $\Gamma_{L}, \Gamma_{R}$, and $x$.

As an example, let us consider quasi-one-dimensional leads, or leads of a quantum wire. The state in lead $\alpha$ is specified by $k=q$ in the case of single conduction channel and by $k=(q, i)$ in the presence of multiple channels, where $q$ is the momentum along the wire and $i$ is the index of the subbands. In the former, $V_{\alpha, k}=V_{\alpha}\left(\varepsilon_{k}\right)$ and $w_{\alpha, k}=w_{\alpha}\left(\varepsilon_{k}\right)$, which yield $\Gamma_{\alpha}(\varepsilon)=\pi \rho_{\alpha}(\varepsilon)\left[V_{\alpha}(\varepsilon)\right]^{2}$ with density of states $\rho_{\alpha}$ in the lead, $x_{\alpha}(\varepsilon)=\pi \rho_{\alpha}(\varepsilon) w_{\alpha}(\varepsilon)$, and $\left|p_{\alpha}\right|=1$ from Eqs. (6) to (8). In the case of multiple channels, $\left|p_{\alpha}\right|<1$, as shown in Sec. V. Note that a similar parameter to $p_{\alpha}$ was introduced in the study on a double quantum dot in parallel and was evaluated for three- or two-dimensional leads with a flat surface [35].

For the multiterminal geometry, lead $\alpha$ is divided into leads $\alpha(1)$ and $\alpha(2)$, as depicted in Fig. 1(b). The Hamiltonian in Eq. (1) is applicable even to this case, in which the summation over $k$ is taken in both lead $\alpha(1)$ [denoted by $\sum_{k}^{(1)}$ ] and lead $\alpha(2)$ [by $\sum_{k}^{(2)}$ ]. We define $\Gamma_{\alpha}^{(j)}$ using Eq. (6) with the summation over $k$ in lead $\alpha(j)$ only

$$
\Gamma_{\alpha}^{(j)}(\varepsilon)=\pi \sum_{k}^{(j)}\left|V_{\alpha, k}\right|^{2} \delta\left(\varepsilon-\varepsilon_{k}\right)
$$

for $\alpha=L, R$ and $j=1,2$. Similarly, we define $x_{\alpha}^{(1)}, x_{\alpha}^{(2)}, p_{\alpha}^{(1)}$, and $p_{\alpha}^{(2)}$. They satisfy the following relations:

$$
\begin{aligned}
\Gamma_{\alpha} & =\Gamma_{\alpha}^{(1)}+\Gamma_{\alpha}^{(2)}, \\
x_{\alpha} & =x_{\alpha}^{(1)}+x_{\alpha}^{(2)} \\
\sqrt{\Gamma_{\alpha} x_{\alpha}} p_{\alpha} & =\sqrt{\Gamma_{\alpha}^{(1)} x_{\alpha}^{(1)}} p_{\alpha}^{(1)}+\sqrt{\Gamma_{\alpha}^{(2)} x_{\alpha}^{(2)}} p_{\alpha}^{(2)} .
\end{aligned}
$$

\section{B. Formulation of electric current}

We formulate the electric current using the Keldysh Green's functions along the lines of Ref. [19] (see Appendix A). For example, the current from lead $L(1)$ in Fig. 1(b) is given by

$$
I_{L}^{(1)}=-e\left\langle\dot{N}_{L}^{(1)}\right\rangle=-\frac{e}{i \hbar}\left\langle\left[N_{L}^{(1)}, H\right]\right\rangle,
$$

where

$$
N_{L}^{(1)}=\sum_{k \sigma}^{(1)} a_{L, k \sigma}^{\dagger} a_{L, k \sigma}
$$

is the number operator for electrons in the lead. In the stationary state, $I_{L}^{(1)}$ is expressed in terms of the retarded Green's function $G_{d, d}^{\mathrm{r}}(\varepsilon)$ and lesser Green's function $G_{d, d}^{<}(\varepsilon)$ of the QD, in Eq. (A22) in Appendix A.

Next, we eliminate $G_{d, d}^{<}(\varepsilon)$ from the expression and write the current using $G_{d, d}^{\mathrm{r}}(\varepsilon)$ only. We restrict ourselves to the 
case of

$$
\mu_{L}^{(1)}=\mu_{L}^{(2)} \equiv \mu_{L}, \quad \mu_{R}^{(1)}=\mu_{R}^{(2)} \equiv \mu_{R}
$$

with $\mu_{L}-\mu_{R}=e V$, to simplify the current expression. Then the current conservation is written as follows in the stationary state:

$$
\begin{aligned}
0= & I_{L}^{(1)}+I_{L}^{(2)}+I_{R}^{(1)}+I_{R}^{(2)} \\
= & \frac{4 e}{h} \int d \varepsilon\left\{-\tilde{\Gamma}\left[\left[f_{L}(\varepsilon)+f_{R}(\varepsilon)\right] \operatorname{Im} G_{d, d}^{\mathrm{r}}(\varepsilon)+\operatorname{Im} G_{d, d}^{<}(\varepsilon)\right]\right. \\
& \left.+\left[f_{L}(\varepsilon)-f_{R}(\varepsilon)\right]\left[-\left(\Gamma_{L}-\Gamma_{R}\right)-4 \frac{\sqrt{\Gamma_{L} \Gamma_{R} x} p_{L} p_{R}}{(1+x)^{2}} \sin \phi+\frac{x(x+3)}{(1+x)^{2}}\left(\Gamma_{L} p_{L}^{2}-\Gamma_{R} p_{R}^{2}\right)\right] \operatorname{Im} G_{d, d}^{\mathrm{r}}(\varepsilon)\right\}
\end{aligned}
$$

where $f_{\alpha}(\varepsilon)=\left[\left(\varepsilon-\mu_{\alpha}\right) /\left(k_{\mathrm{B}} T\right)+1\right]^{-1}$ is the Fermi distribution function in lead $\alpha(1)$ or $\alpha(2)[\tilde{\Gamma}$ will be given in Eq. (24)]. Using Eq. (18), we eliminate $G_{d, d}^{<}(\varepsilon)$ from the current expression, e.g., Eq. (A22) for $I_{L}^{(1)}$.

\section{Electric current in two-terminal systems}

Now we present the expression for the electric current in the two-terminal systems. The current from the left lead $I_{L}[=$ $I_{L}^{(1)}+I_{L}^{(2)}$ using the results in Appendix A] is expressed as

$$
I_{L}=\frac{2 e}{h} \int d \varepsilon\left[f_{L}(\varepsilon)-f_{R}(\varepsilon)\right] T(\varepsilon)
$$

with the transmission probability

$$
T(\varepsilon)=\frac{4 x}{(1+x)^{2}}+8 \frac{1-x}{(1+x)^{3}} \sqrt{\Gamma_{L} \Gamma_{R} x} p_{L} p_{R} \cos \phi \operatorname{Re} G_{d, d}^{\mathrm{r}}(\varepsilon)+\frac{4 C_{1}}{(1+x)^{3} \tilde{\Gamma}} \operatorname{Im} G_{d, d}^{\mathrm{r}}(\varepsilon) .
$$

Here, the coefficient $C_{1}$ is given by

$$
\begin{aligned}
C_{1}= & \frac{x^{3}}{1+x}\left[\left(\Gamma_{L} p_{L}^{2}\right)^{2}+\left(\Gamma_{R} p_{R}^{2}\right)^{2}\right]+x(1-x)\left[\left(\Gamma_{L} p_{L}\right)^{2}+\left(\Gamma_{R} p_{R}\right)^{2}\right] \\
& -\Gamma_{L} \Gamma_{R}\left[(1+x)^{3}+\frac{4 x}{1+x}\left(p_{L} p_{R}\right)^{2} \sin ^{2} \phi+\frac{x^{2}\left(x^{2}+4 x+9\right)}{1+x}\left(p_{L} p_{R}\right)^{2}-x\left(x^{2}+3 x+4\right)\left(p_{L}^{2}+p_{R}^{2}\right)\right] .
\end{aligned}
$$

Note that (i) for $p_{L}=p_{R}=1$, where a single conduction channel is effective in each lead, Eq. (20) coincides with the current expression derived in Ref. [19]. (ii) For $p_{L}=p_{R}=0$, the transmission probability is given by

$$
T(\varepsilon)=\frac{4 x}{(1+x)^{2}}-\frac{4 \Gamma_{L} \Gamma_{R}}{\Gamma_{L}+\Gamma_{R}} \operatorname{Im} G_{d, d}^{\mathrm{r}}(\varepsilon) .
$$

This is the summation of the transmission probability through the upper arm, $T_{\text {upper }}$ in Eq. (9), and that through the QD, indicating no interference effect between the two paths in the QD interferometer.

For multiterminal systems, the current is expressed in terms of the retarded Green's function $G_{d, d}^{\mathrm{r}}(\varepsilon)$ in a similar way. The expression is given in Eqs. (A29) and (A30) in Appendix A.

\section{Exact calculation for Kondo effect}

In the absence of Coulomb interaction, $U=0$, the retarded Green's function of the QD is given by $G_{d, d}^{\mathrm{r}}(\varepsilon)=1 /$ $\left(\varepsilon-\varepsilon_{d}-\Sigma_{d}\right)$, where the self-energy by the tunnel couplings is

$$
\Sigma_{d}=-\frac{2 \sqrt{\Gamma_{L} \Gamma_{R} x}}{1+x} p_{L} p_{R} \cos \phi-i \tilde{\Gamma}
$$

with an effective linewidth

$$
\tilde{\Gamma}=\Gamma_{L}\left(1-\frac{x}{1+x} p_{L}^{2}\right)+\Gamma_{R}\left(1-\frac{x}{1+x} p_{R}^{2}\right) .
$$

This expression is common to two- and multiterminal systems.

In the presence of $U, G_{d, d}^{\mathrm{r}}(\varepsilon)$ is evaluated exactly in the following way. The Green's function at $U=0$ indicates that our models are equivalent to the situation in which a QD with an energy level

$$
\tilde{\varepsilon}_{d}(\phi)=\varepsilon_{d}-\frac{2 \sqrt{\Gamma_{L} \Gamma_{R} x}}{1+x} p_{L} p_{R} \cos \phi
$$

is connected to a lead with linewidth $\tilde{\Gamma}$, as shown in Appendix B. In the Fermi liquid theory, the Green's function is written as

$$
G_{d, d}^{\mathrm{r}}(0)=\frac{z}{-\tilde{\varepsilon}_{d}^{*}+i z \tilde{\Gamma}}=\frac{\tilde{\Gamma}^{*}}{\tilde{\Gamma}} \frac{1}{-\tilde{\varepsilon}_{d}^{*}+i \tilde{\Gamma}^{*}},
$$

at $\varepsilon=E_{\mathrm{F}}=0$, where $\tilde{\varepsilon}_{d}^{*}$ is the renormalized value of $\tilde{\varepsilon}_{d}(\phi)$ in Eq. (25), $\tilde{\Gamma}^{*}=z \tilde{\Gamma}$ is that of $\tilde{\Gamma}$ in Eq. (24), and $z$ is a factor of wave-function renormalization by the electron-electron interaction $U$ [36-38]. Since the phase shift $\theta_{\mathrm{QD}}$ at the $\mathrm{QD}$ is given by $\tan \theta_{\mathrm{QD}}=\tilde{\Gamma}^{*} / \tilde{\varepsilon}_{d}^{*}$, the Green's function is determined 
by $\theta_{\mathrm{QD}}$ as

$$
G_{d, d}^{\mathrm{r}}(0)=\frac{-1}{\tilde{\Gamma}} e^{i \theta_{\mathrm{QD}}} \sin \theta_{\mathrm{QD}} .
$$

$\theta_{\mathrm{QD}}$ is related to the electron number per spin in the QD through the Friedel sum rule, $\theta_{\mathrm{QD}}=\pi\left\langle n_{\sigma}\right\rangle .\left\langle n_{\sigma}\right\rangle$ is evaluated at temperature $T=0$ using the Bethe ansatz exact solution $[39,40]$. Hence we can precisely calculate $G_{d, d}^{\mathrm{r}}(0)$ and thus the conductance $G=d I_{L} / d V(V \rightarrow 0)$ at $T=0$ in the presence of $U$.

It is worth mentioning that the effective energy level $\tilde{\varepsilon}_{d}(\phi)$ in the QD gives rise to the $\phi$-dependent Kondo temperature [20]. It is written as

$k_{\mathrm{B}} T_{\mathrm{K}}(\phi) \approx D\left[\frac{\tilde{\Gamma} U}{\left|\tilde{\varepsilon}_{d}(\phi)\right|\left[\tilde{\varepsilon}_{d}(\phi)+U\right]}\right]^{1 / 2} e^{-\pi\left|\tilde{\varepsilon}_{d}(\phi)\right|\left[\tilde{\varepsilon}_{d}(\phi)+U\right] /(2 \tilde{\Gamma} U)}$,

with $D$ being the bandwidth [36,41] although $T_{\mathrm{K}}(\phi)$ is irrelevant to our study on the transport properties at $T=0$.

\section{CALCULATED RESULTS IN TWO-TERMINAL GEOMETRY}

In this section, we present the calculated results for the two-terminal system, paying attention to the shape of a conductance peak as a function of energy level $\varepsilon_{d}$ in the QD. We find that parameters $p_{L}$ and $p_{R}$ are relevant in both the cases of $U=0$ and $U \neq 0$.

\section{A. Fano versus Breit-Wigner resonance}

We begin with the case of no electron-electron interaction in the $\mathrm{QD}, U=0$. Figure 2 shows the conductance $G$ at $T=0$ as a function of energy level $\varepsilon_{d}$ in the QD for (a) $p_{L}=p_{R}=1$, (b) 0.75 , and (c) 0.5 . The $\mathrm{AB}$ phase is $\phi=0$ (solid line), $\pm \pi / 2$ (broken line), and $\pi$ (dotted line). $G(\phi)=G(-\phi)$ holds by the Onsager's reciprocal theorem.

In Fig. 2(a) with $p_{L}=p_{R}=1$, the conductance $G$ shows an asymmetric resonant shape with dip and peak in the absence of magnetic field $(\phi=0)$. This is known as the Fano resonance, which is ascribable to the interference between the tunneling through a discrete level and that through continuous states $[6,7]$. A magnetic field changes the resonant shape to be symmetric at $\phi= \pm \pi / 2$ and asymmetric with peak and dip at $\phi=\pi$. This Fano resonance is characterized by a complex Fano factor [5]. Indeed the conductance can be analytically expressed [42] in the form of

$$
G=\frac{2 e^{2}}{h} \frac{4 x}{(1+x)^{2}} \frac{|e+q|^{2}}{e^{2}+1}
$$

with $e=\left[\varepsilon_{d}-\tilde{\varepsilon}_{d}(\phi)\right] / \tilde{\Gamma}$, where $\tilde{\varepsilon}_{d}(\phi)=\varepsilon_{d}-2 \sqrt{\Gamma_{L} \Gamma_{R} x} \cos \phi /$ $(1+x)$ and $\tilde{\Gamma}=\left(\Gamma_{L}+\Gamma_{R}\right) /(1+x)$ [Eqs. (25) and (24) for $\left.p_{L}=p_{R}=1\right]$. The complex Fano factor is given by

$$
q=\frac{\sqrt{\Gamma_{L} \Gamma_{R}}}{\tilde{\Gamma} \sqrt{x}}\left(\frac{1-x}{1+x} \cos \phi-i \sin \phi\right) .
$$

With a decrease in $p_{L}$ and $p_{R}$, the conductance peak becomes more symmetric and its $\phi$-dependence is less prominent, as shown in Figs. 2(b) and 2(c). The shape of

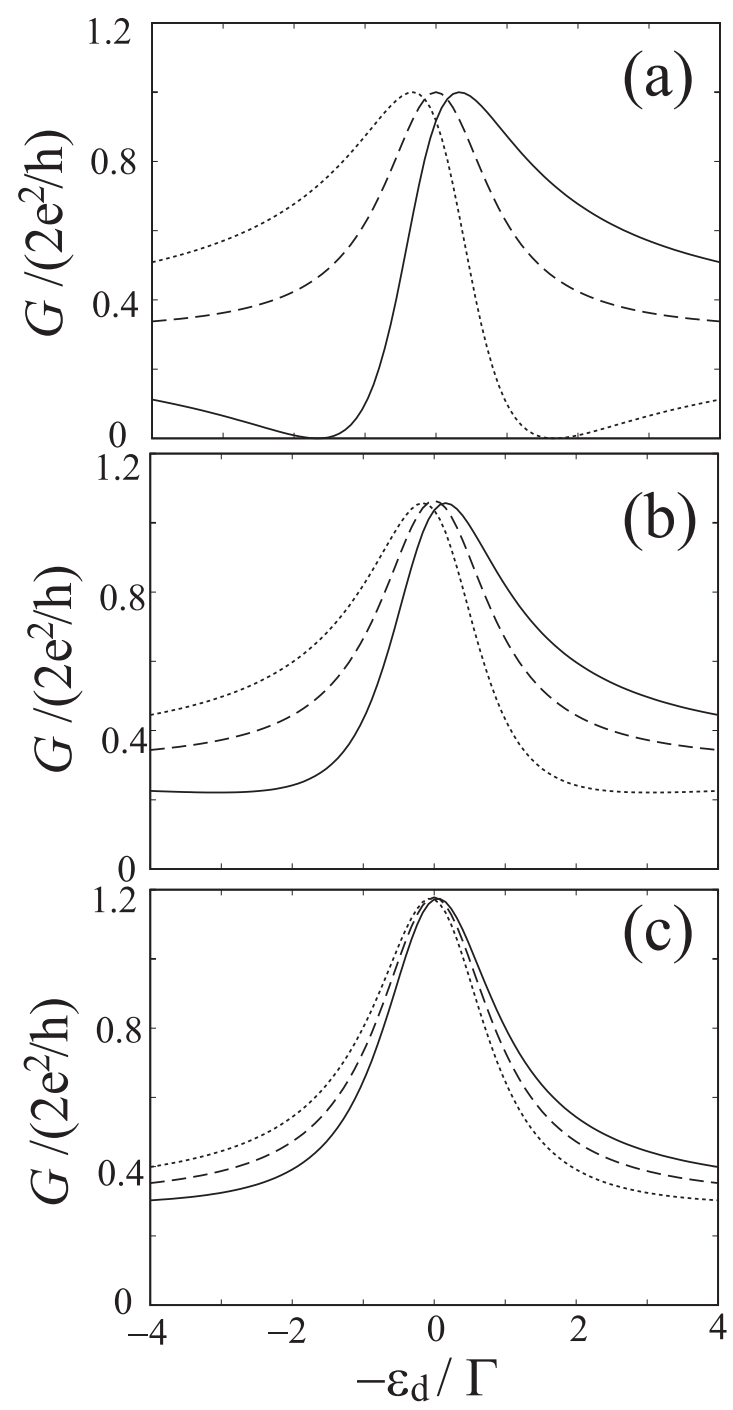

FIG. 2. Calculated results for the conductance $G$ in the twoterminal system in the absence of $U . G$ at temperature $T=0$ is plotted as a function of energy level $\varepsilon_{d}$ in the quantum dot. $\Gamma_{L}=$ $\Gamma_{R}=\Gamma / 2, x=0.09\left(x_{L}=x_{R}=0.3\right)$, and (a) $p_{L}=p_{R}=1$, (b) 0.75 , and (c) 0.5 . The $\mathrm{AB}$ phase for the magnetic flux penetrating the ring is $\phi=0$ (solid line), $\phi= \pm \pi / 2$ (broken line), and $\phi=\pi$ (dotted line).

conductance peak is closer to that of the Lorentzian function of Breit-Wigner resonance as $p_{L}$ and $p_{R}$ go to zero.

Note that the conductance $G$ can exceed unity in units of $2 e^{2} / h$ when $p_{L}, p_{R}<1$, reflecting the multiple conduction channels in the leads. See Eq. (22) in the limit of $p_{L}=p_{R}=$ 0 : The upper limit of $G /\left(2 e^{2} / h\right)$ is the sum of the transmission probability through the QD (unity if $\Gamma_{L}=\Gamma_{R}$ ) and that through the upper arm, $T_{\text {upper }}$ in Eq. (9).

\section{B. Fano-Kondo resonance versus Kondo plateau}

In the presence of $U$, the Kondo effect is exactly taken into account in the evaluation of the conductance at $T=0$, as described in the previous section. In Fig. 3, the conductance $G$ is shown as a function of energy level $\varepsilon_{d}$ in the QD, for $U / \Gamma=8$ and $\Gamma_{L}=\Gamma_{R}=\Gamma / 2$; (a) $p_{L}=p_{R}=1$, (b) 0.75 , and 

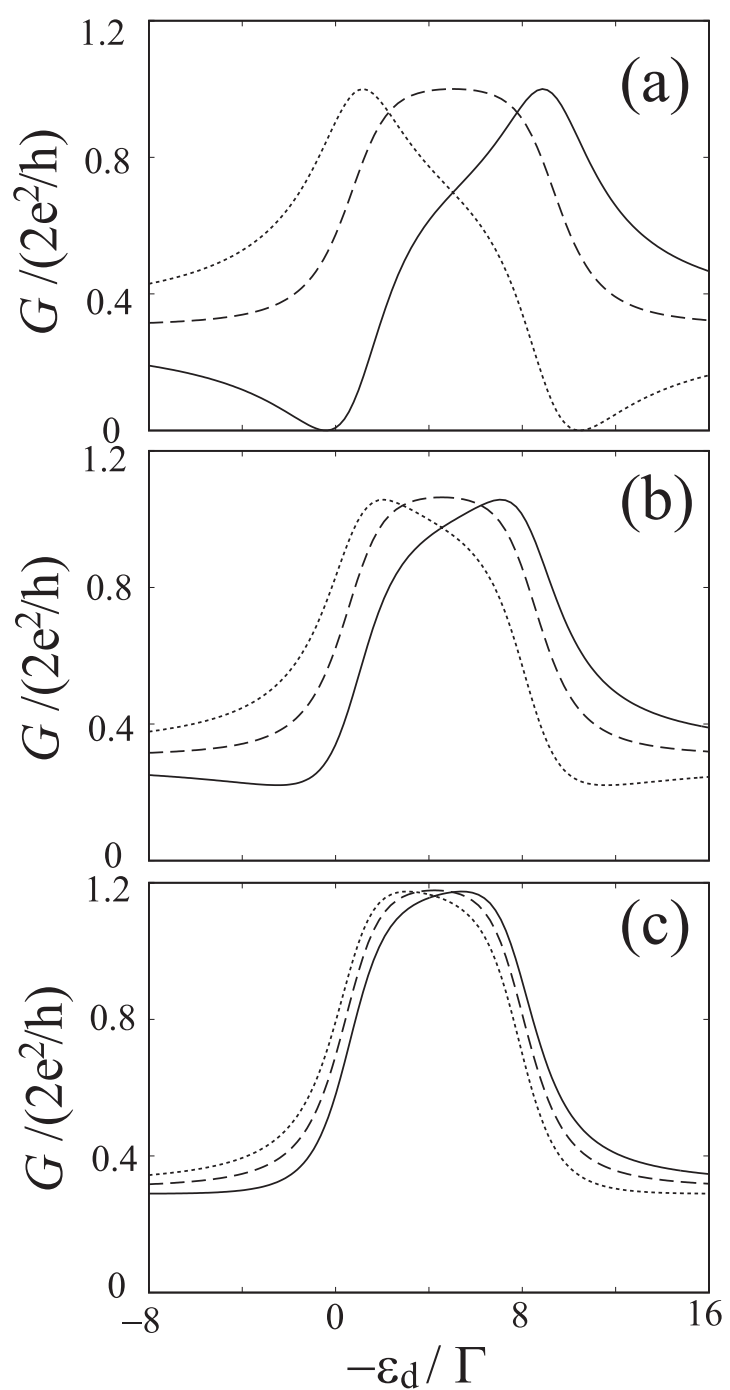

FIG. 3. Calculated results for the conductance $G$ in the twoterminal system in the presence of $U . G$ at temperature $T=0$ is plotted as a function of energy level $\varepsilon_{d}$ in the quantum dot. $\Gamma_{L}=$ $\Gamma_{R}=\Gamma / 2, x=0.09\left(x_{L}=x_{R}=0.3\right)$, and (a) $p_{L}=p_{R}=1$, (b) 0.75 , and (c) 0.5. $U=8 \Gamma$. The $\mathrm{AB}$ phase for the magnetic flux penetrating the ring is $\phi=0$ (solid line), $\phi= \pm \pi / 2$ (broken line), and $\phi=\pi$ (dotted line).

(c) 0.5. The AB phase is $\phi=0$ (solid line), $\pm \pi / 2$ (broken line), and $\pi$ (dotted line).

For $p_{L}=p_{R}=1, G$ behaves as a "Fano-Kondo resonance" proposed by Hofstetter et al. [19], which stems from an interplay between the Kondo resonance $\left(G \sim 2 e^{2} / h\right.$ at $-U<$ $\left.\varepsilon_{d}<0\right)$ and the Fano resonance. When $\phi=0(\pi), G$ shows a dip and peak (peak and dip) with a gradual slope around the center of the Kondo valley, i.e., Coulomb blockade regime with a spin $1 / 2$ in the QD. When $\phi=\pi / 2, G$ is almost constant at $2 e^{2} / h$ in the Kondo valley and symmetric with respect to the valley center.

With decreasing $p_{L}$ and $p_{R}$, the asymmetric shape of the Fano-Kondo resonance changes to a conductance plateau, the so-called Kondo plateau, in the Kondo valley: $G \rightarrow 2 e^{2} / h+$ $T_{\text {upper }}$ as $p_{L, R} \rightarrow 0$ when $\Gamma_{L}=\Gamma_{R}$. Besides, $G$ is less dependent on $\phi$.

\section{CALCULATED RESULTS IN THREE-TERMINAL GEOMETRY}

In this section, we examine a three-terminal system to discuss the measurement of transmission phase shift through the QD by a "double-slit interference experiment." We assume two leads $R(1)$ and $R(2)$ on the right side and a single lead $L$ on the left side in Fig. 1(b). We evaluate the conductance from lead $L$ to $R(1)$ or to $R(2)$,

$$
G^{(1)}=-\frac{d I_{R}^{(1)}}{d V}, \quad G^{(2)}=-\frac{d I_{R}^{(2)}}{d V},
$$

for $e V=\mu_{L}-\mu_{R} \rightarrow 0\left(\mu_{R}^{(1)}=\mu_{R}^{(2)}=\mu_{R}\right)$ at $T=0$, as a function of AB phase $\phi$. We define the measured phase shift by the $\mathrm{AB}$ phase $\phi_{\max }$ at which the conductance $G^{(1)}(\phi)$ is maximal.

As an intrinsic transmission phase shift through the QD, we introduce $\theta_{\mathrm{QD}}^{(0)}$ and $\theta_{\mathrm{QD}}$ by

$$
\begin{aligned}
\tan \theta_{\mathrm{QD}}^{(0)} & =\frac{\Gamma_{L}+\Gamma_{R}}{\varepsilon_{d}-E_{\mathrm{F}}}, \\
\tan \theta_{\mathrm{QD}} & =\frac{\tilde{\Gamma}}{\tilde{\varepsilon}_{d}(\phi)-E_{\mathrm{F}}},
\end{aligned}
$$

respectively, in the absence of $U \cdot \theta_{\mathrm{QD}}^{(0)}$ is the phase shift through the QD without the upper arm of the ring, whereas $\theta_{\mathrm{QD}}$ satisfies the Friedel sum rule $\theta_{\mathrm{QD}}=\pi\left\langle n_{\sigma}\right\rangle$ for the QD embedded in the ring. This last depends on the AB phase $\phi$ for the magnetic flux penetrating the ring. In the next subsection, we derive an analytical relation between the measured phase $\phi_{\max }$ and $\theta_{\mathrm{QD}}^{(0)}$ in Eq. (32) in the absence of $U$.

In Secs. IV.B and C, we examine two specific models depicted in Fig. 4. In Fig. 4(a), leads $L$ and $R(1)$ are connected
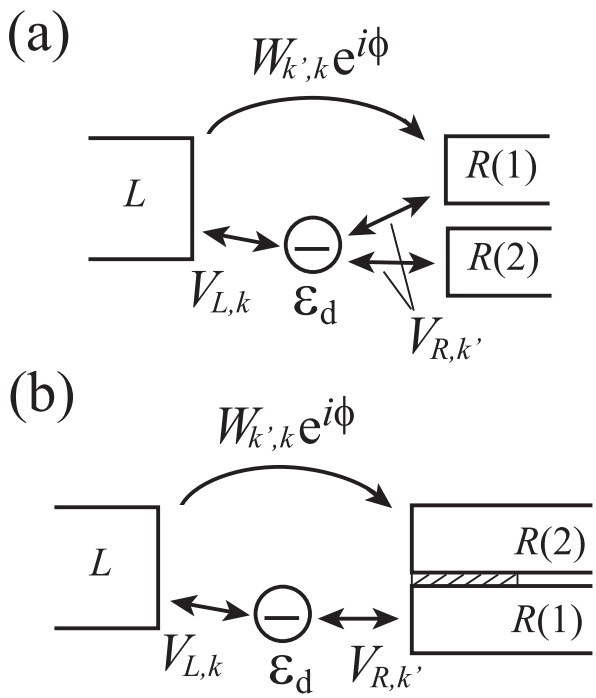

FIG. 4. Two specific models for the three-terminal system. (a) Leads $L$ and $R(1)$ are connected to both the quantum dot and upper arm of the ring, whereas lead $R(2)$ is connected to the quantum dot only. (b) Leads $R(1)$ and $R(2)$ are quantum wires which are tunnel-coupled to each other at the hatched region. Lead $L$ is connected to both the quantum dot and upper arm of the ring, whereas lead $R(1)[R(2)]$ is connected to the quantum dot [upper arm of the ring] only at the end of the leads. 
to both the QD and upper arm of the ring, whereas lead $R(2)$ is connected to the QD only. We vary the strength of tunnel coupling to lead $R(2), \Gamma_{R}^{(2)}$, to investigate a crossover from two- to three-terminal phase measurement. In Fig. 4(b), we model the experimental situation by Takada et al., in which leads $R(1)$ and $R(2)$ are partly coupled quantum wires $[8,16,17]$.

\section{A. Measured phase shift for $\boldsymbol{U}=\mathbf{0}$}

For the three-terminal model in Fig. 1(b) with leads $L$, $R(1)$, and $R(2)$, we introduce the following dimensionless parameters:

$$
\gamma_{R}^{(j)}=\frac{\Gamma_{R}^{(j)}}{\Gamma_{R}}, \quad y_{R}^{(j)}=\frac{x_{R}^{(j)}}{x_{R}}, \quad q_{R}^{(j)}=\frac{\sqrt{\Gamma_{R}^{(j)} x_{R}^{(j)}} p_{R}^{(j)}}{\sqrt{\Gamma_{R} x_{R}} p_{R}}
$$

for $j=1$ and 2. They are the ratios of contribution from lead $R(j)$ to $\Gamma_{R}, x_{R}$, and $\sqrt{\Gamma_{R} x_{R}} p_{R}$, respectively, and satisfy the relations of $\gamma_{R}^{(1)}+\gamma_{R}^{(2)}=y_{R}^{(1)}+y_{R}^{(2)}=q_{R}^{(1)}+q_{R}^{(2)}=1$.

In the absence of $U$, Eqs. (A29) and (A30) yield the conductance in the form of

$$
\begin{aligned}
G^{(1)}= & \frac{2 e^{2}}{h} \frac{1}{\left[E_{\mathrm{F}}-\tilde{\varepsilon}_{d}(\phi)\right]^{2}+\tilde{\Gamma}^{2}} \\
& \times\left[\frac{8 \sqrt{\Gamma_{L} \Gamma_{R} x} p_{L} p_{R}}{(1+x)^{2}} F(\phi)+(\phi \text {-indep. terms })\right],
\end{aligned}
$$

where

$$
\begin{aligned}
F(\phi)=q_{R}^{(1)}\left(\varepsilon-\varepsilon_{d}\right) \cos \phi & +\left[x\left(q_{R}^{(1)}-y_{R}^{(1)}\right) \Gamma_{L}\left(1-p_{L}^{2}\right)\right. \\
+ & \left.\left(\gamma_{R}^{(1)}-q_{R}^{(1)}\right) \Gamma_{R}\right] \sin \phi .
\end{aligned}
$$

If we neglect the $\phi$-dependence in $\tilde{\varepsilon}_{d}(\phi)$ in the denominator in Eq. (35), the measured phase $\phi_{\max }$ is given by

$$
\begin{aligned}
\tan \phi_{\max }= & \frac{x\left(y_{R}^{(1)}-q_{R}^{(1)}\right) \Gamma_{L}\left(1-p_{L}^{2}\right)+\left(q_{R}^{(1)}-\gamma_{R}^{(1)}\right) \Gamma_{R}}{q_{R}^{(1)}\left(\Gamma_{L}+\Gamma_{R}\right)} \\
& \times \tan \theta_{\mathrm{QD}}^{(0)},
\end{aligned}
$$

where $\theta_{\mathrm{QD}}^{(0)}$ is defined in Eq. (32). This is an approximate formula for the relation between the measured value and intrinsic value of the transmission phase shift through the QD.

In the two-terminal geometry, lead $R(2)$ is absent and thus $\gamma_{R}^{(1)}=y_{R}^{(1)}=q_{R}^{(1)}=1$. Then Eq. (37) yields $\tan \phi_{\max }=0$, i.e., $\phi_{\max }=0$ or $\pi$ in accordance with the Onsager's reciprocal theorem.

\section{B. Model in Fig. 4(a) with $U=0$}

To elucidate a crossover from two- to three-terminal measurement of the transmission phase shift through the QD, we examine the model depicted in Fig. 4(a) with $U=0$. In this model, leads $L$ and $R(1)$ are connected to both the QD and upper arm of the ring, whereas lead $R(2)$ is connected to the QD only. From $x_{R}^{(2)}=0$ and $\Gamma_{R}=\Gamma_{R}^{(1)}+\Gamma_{R}^{(2)}$, dimensionless parameters in the previous subsection become $\gamma_{R}^{(1)}=\Gamma_{R}^{(1)} / \Gamma_{R}$ and $y_{R}^{(1)}=q_{R}^{(1)}=1$. In the QD, the effective energy level and linewidth are $\tilde{\varepsilon}_{d}(\phi)=\varepsilon_{d}-2 \sqrt{\Gamma_{L} \Gamma_{R}^{(1)} x} p_{L} p_{R}^{(1)} \cos \phi /(1+x)$ and $\tilde{\Gamma}=\Gamma_{L}\left[1-x p_{L}^{2} /(1+x)\right]+\Gamma_{R}^{(1)}\left[1-x p_{R}^{(1) 2} /(1+x)\right]+\Gamma_{R}^{(2)}$,
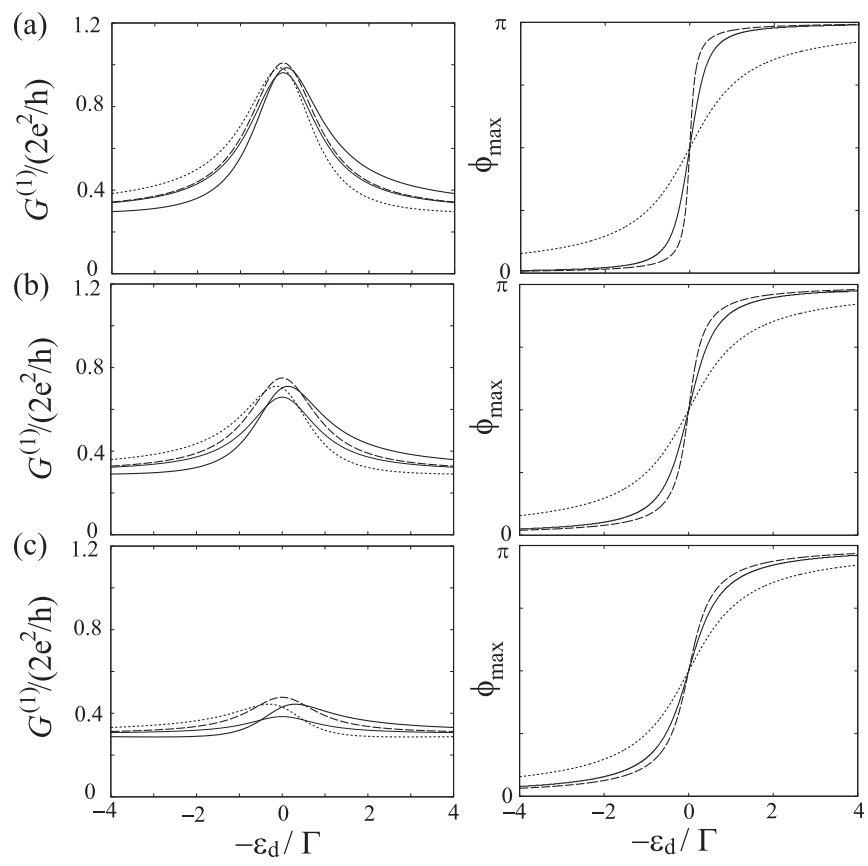

FIG. 5. Calculated results for the three-terminal model depicted in Fig. 4(a) in the absence of $U$. In the left panels, the conductance $G^{(1)}$ to lead $R(1)$ at temperature $T=0$ is plotted as a function of energy level $\varepsilon_{d}$ in the quantum dot. $\Gamma_{L}=\Gamma_{R}=\Gamma / 2, x=0.09$ $\left(x_{L}=x_{R}=0.3\right)$, and $p_{L}=p_{R}=0.5$. The tunnel coupling to lead $R(2)$ is increased from (a) to (c): (a) $\Gamma_{R}^{(2)} / \Gamma_{R}=0.2$, (b) 0.5 , and (c) 0.8 with $\Gamma_{R}^{(1)}+\Gamma_{R}^{(2)}=\Gamma_{R}$. The AB phase for the magnetic flux penetrating the ring is $\phi=0$ (solid line), $\phi=\pi / 2$ (broken line), $\phi=\pi$ (dotted line), and $\phi=-\pi / 2$ (thin solid line). In the right panels, the measured phase shift $\phi_{\max }$ is plotted as a function of $\varepsilon_{d}$ (solid line), which is numerically evaluated as the $\mathrm{AB}$ phase when $G^{(1)}(\phi)$ is maximal. $\phi_{\max }$ given by the formula in Eq. (38) is plotted by broken line, whereas the transmission phase shift $\theta_{\mathrm{QD}}^{(0)}$ through the quantum dot without the upper arm of the ring is plotted by dotted line.

respectively. Equation (37) yields an approximate relation of

$$
\tan \phi_{\max }=\frac{\Gamma_{R}^{(2)}}{\Gamma_{L}+\Gamma_{R}^{(1)}+\Gamma_{R}^{(2)}} \tan \theta_{\mathrm{QD}}^{(0)},
$$

which indicates that the measured phase shift $\phi_{\max }$ approaches the intrinsic phase shift $\theta_{\mathrm{QD}}^{(0)}$ with an increase in $\Gamma_{R}^{(2)}$.

Figure 5 presents the calculated results for the model in Fig. 4(a). In the left panels, the conductance $G^{(1)}$ to lead $R(1)$ at $T=0$ is plotted as a function of energy level $\varepsilon_{d}$ in the QD. $\Gamma_{L}=\Gamma_{R}=\Gamma / 2$ and (a) $\Gamma_{R}^{(2)} / \Gamma_{R}=0.2$, (b) 0.5 , and (c) 0.8. For small $\Gamma_{R}^{(2)} / \Gamma_{R}$ [Fig. 5(a)], $G^{(1)}$ is almost the same at $\phi= \pm \pi / 2$ corresponding to the Onsager's reciprocal theorem in the two-terminal system. With increasing $\Gamma_{R}^{(2)} / \Gamma_{R}$ [Figs. 5(b) and 5(c)], the deviation from the theorem becomes more prominent. The peak height of $G^{(1)}$ is reduced by stronger tunnel coupling to lead $R(2)$.

The right panels in Fig. 5 show $\phi_{\max }$ that is numerically evaluated from $G^{(1)}(\phi)$, as a function of $\varepsilon_{d}$ (solid lines). The intrinsic phase shift $\theta_{\mathrm{QD}}^{(0)}$ in Eq. (32) is plotted by dotted lines. Broken lines show $\phi_{\max }$ in Eq. (38), indicating that the formula is a good approximation to estimate $\phi_{\max }$ from 


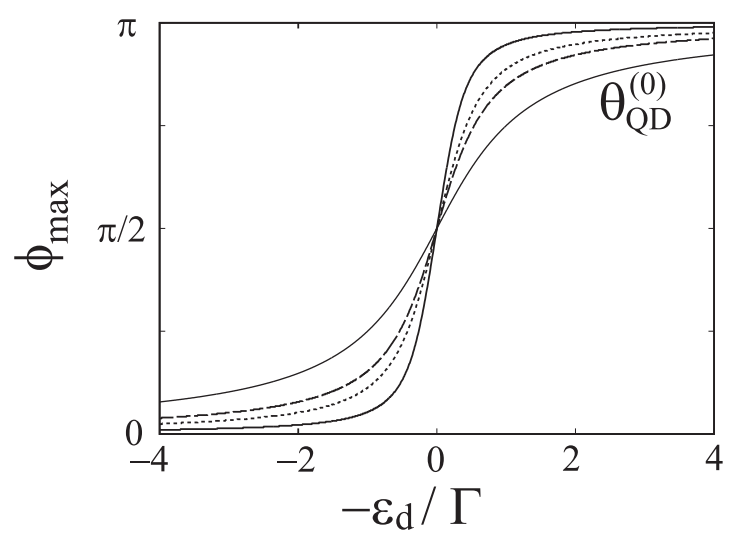

FIG. 6. Measured phase shift $\phi_{\max }$ as a function of energy level $\varepsilon_{d}$ in the quantum dot, in the three-terminal model depicted in Fig. 4(a) in the absence of $U$. The data for $\phi_{\max }$ are the same as in the right panels in Fig. 5. $\Gamma_{R}^{(2)} / \Gamma_{R}=0.2$ (solid line), 0.5 (dotted line), and 0.8 (broken line). A thin solid line indicates the transmission phase shift $\theta_{\mathrm{QD}}^{(0)}$ through the quantum dot without the upper arm of the ring.

$\theta_{\mathrm{QD}}^{(0)}$. In Fig. 5(a) with $\Gamma_{R}^{(2)} / \Gamma_{R}=0.2, \phi_{\max }$ changes almost abruptly from zero to $\pi$ around $\varepsilon_{d}=E_{\mathrm{F}}=0$, which is close to the behavior in the two-terminal system. For larger $\Gamma_{R}^{(2)} / \Gamma_{R}$, $\phi_{\max }$ changes more gradually with $\varepsilon_{d}$ and closer to the intrinsic phase shift $\theta_{\mathrm{QD}}^{(0)}$ although $\phi_{\max }$ does not go to $\theta_{\mathrm{QD}}^{(0)}$ as $\Gamma_{R}^{(2)} / \Gamma_{R} \rightarrow 1$ under the condition of $\Gamma_{L}=\Gamma_{R}$.

To illustrate the crossover from the two- to three-terminal phase measurement, we replot $\phi_{\max }$ for three values of $\Gamma_{R}^{(2)} / \Gamma_{R}$ in a graph in Fig. 6.

\section{Model in Fig. 4(b)}

Now we study the model shown in Fig. 4(b) to examine the experimental situation using partly coupled quantum wires to form a mesoscopic ring $[8,16,17]$. We assume that leads $R(1)$ and $R(2)$ consist of two equivalent wires $a$ and $b$ of single conduction channel. They are tunnel-coupled to each other in the vicinity of their edges, which mixes states $\left|a, k^{\prime}\right\rangle$ in lead $a$ and $\left|b, k^{\prime}\right\rangle$ in lead $b$. As a result, the edge states in leads $R(1)$ and $R(2)$ are given by

$$
\begin{aligned}
& \left|\psi_{R k^{\prime}}^{(1)}\right\rangle=\alpha_{R}\left|a, k^{\prime}\right\rangle+\beta_{R}\left|b, k^{\prime}\right\rangle, \\
& \left|\psi_{R k^{\prime}}^{(2)}\right\rangle=\beta_{R}\left|a, k^{\prime}\right\rangle-\alpha_{R}\left|b, k^{\prime}\right\rangle,
\end{aligned}
$$

respectively, with real coefficients $\alpha_{R}$ and $\beta_{R}\left(\alpha_{R}^{2}+\beta_{R}^{2}=1\right)$. Far from the edges, $\left|\psi_{R k^{\prime}}^{(1)}\right\rangle \rightarrow\left|a, k^{\prime}\right\rangle$ in lead $R(1)$ and $\left|\psi_{R k^{\prime}}^{(2)}\right\rangle \rightarrow\left|b, k^{\prime}\right\rangle$ in lead $R(2)$ in an asymptotic way.

As shown in Fig. 4(b), $\left|\psi_{R k^{\prime}}^{(1)}\right\rangle$ in Eq. (39) is coupled to the QD while $\left|\psi_{R k^{\prime}}^{(2)}\right\rangle$ in Eq. (40) is connected to the upper arm of the ring. In the tunnel Hamiltonian $H_{T}$ in Eq. (4), $V_{R, k^{\prime}}=V_{R} \alpha_{R}$ and $\sqrt{w_{R, k^{\prime}}}=\sqrt{w_{R}} \beta_{R}$ when state $k^{\prime}$ belongs to lead $R(1)$ while $V_{R, k^{\prime}}=V_{R} \beta_{R}$ and $\sqrt{w_{R, k^{\prime}}}=-\sqrt{w_{R}} \alpha_{R}$ when state $k^{\prime}$ belongs to lead $R(2)$. Thus $\Gamma_{R}^{(1)}=\alpha_{R}^{2} \Gamma_{R}, \Gamma_{R}^{(2)}=\beta_{R}^{2} \Gamma_{R}$, $x_{R}^{(1)}=\beta_{R}^{2} x_{R}$, and $x_{R}^{(2)}=\alpha_{R}^{2} x_{R}$.

In this model, $p_{R}=0\left(p_{R}^{(1)}=1, p_{R}^{(2)}=-1\right)$ as explained in Appendix $\mathrm{C}$ and in consequence $\tilde{\varepsilon}_{d}(\phi)=\varepsilon_{d}$ in Eq. (25). For
$U=0$, Eq. (37) exactly holds, which yields

$$
\tan \phi_{\max }=\frac{-x \Gamma_{L}\left(1-p_{L}^{2}\right)+\Gamma_{R}}{\Gamma_{L}+\Gamma_{R}} \tan \theta_{\mathrm{QD}}^{(0)} .
$$

In addition, the phase shift $\theta_{\mathrm{QD}}$ can be defined independently of $\phi$, which satisfies the Friedel sum rule in the QD embedded in the ring [see Eq. (33) in the case of $U=0$ ]. For both $U=0$ and $U \neq 0$, we obtain an exact relation of

$$
\tan \phi_{\max }=\frac{-x \Gamma_{L}\left(1-p_{L}^{2}\right)+\Gamma_{R}}{\tilde{\Gamma}} \tan \theta_{\mathrm{QD}}
$$

with $\tilde{\Gamma}=\Gamma_{L}\left[1-x p_{L}^{2} /(1+x)\right]+\Gamma_{R} \cdot \tan \theta_{\mathrm{QD}}=\tilde{\Gamma} / \varepsilon_{d}$ in the absence of $U$ and $\tan \theta_{\mathrm{QD}}=\tilde{\Gamma}^{*} / \tilde{\varepsilon}_{d}^{*}$ in the presence of $U$ when $E_{\mathrm{F}}=0$. Neither Eq. (41) nor Eq. (42) depend on $\alpha_{R}$ and $\beta_{R}$.

We show the calculated results for $U=0$ in Fig. 7. In Figs. 7(a) and 7(b), the conductance $G^{(1)}$ is shown as a function of energy level $\varepsilon_{d}$ in the QD, for (a) $\beta_{R}^{2}=0.1$ and (b) 0.5 . The height of $G^{(1)}$ depends on $\phi$ more largely in Fig. 7(b) than in Fig. 7(a) though $\phi_{\max }$ does not depend on $\beta_{R}$.

Figure 7(c) plots $\phi_{\max }$ that is numerically evaluated from $G^{(1)}(\phi)$. It changes smoothly from zero to $\pi$ via $\pi / 2$ at $\varepsilon_{d}=$ 0 . $\phi_{\max }$ quantitatively deviates from $\theta_{\mathrm{QD}}^{(0)}$ and $\theta_{\mathrm{QD}}$ (dotted and thin solid lines). Their relations are exactly given by Eqs. (41) and (42).

It should be mentioned that the sum of the currents to leads $R(1)$ and $R(2), I_{R}^{(1)}+I_{R}^{(2)}$, does not depend on the AB phase $\phi$, reflecting $p_{R}=0$ in this model (see Appendix C). Therefore, the $\mathrm{AB}$ oscillation of $G^{(1)}(\phi)$ is out-of-phase to that of $G^{(2)}(\phi)$, as indicated in the insets in Fig. 7. $\phi_{\max }$ evaluated from $G^{(1)}$ behaves similarly to $\theta_{\mathrm{QD}}^{(0)}$, while that from $G^{(2)}$ similarly to $-\theta_{\mathrm{QD}}^{(0)}$, irrespective of the absence or presence of $U$ This agrees with the experimental observation by Takada et al. $[8,16]$.

Finally, the measured phase is discussed in the Kondo regime with $U \neq 0$. In Fig. 8, we plot $\phi_{\max }$ that is numerically evaluated from $G^{(1)}$, as a function of energy level $\varepsilon_{d}$ in the QD; (a) $U / \Gamma=8$ and (b) 16 with $\Gamma_{L}=\Gamma_{R}=\Gamma / 2$. In the Kondo valley $\left(-U<\varepsilon_{d}<0\right)$, the phase locking at $\pi / 2$ is observable by a "double-slit experiment" using the QD interferometer. We calculate the intrinsic phase shift $\theta_{\mathrm{QD}}$ using the Friedel sum rule $\theta_{\mathrm{QD}}=\pi\left\langle n_{\sigma}\right\rangle$, where $\left\langle n_{\sigma}\right\rangle$ is given by the Bethe ansatz exact solution (dotted line). $\phi_{\max }$ and $\theta_{\mathrm{QD}}$ are related to each other by Eq. (42). The phase locking seems smeared in the curve of the measured phase shift $\phi_{\max }$, in comparison with the intrinsic phase shift $\theta_{\mathrm{QD}}$.

\section{DISCUSSION}

In our models shown in Figs. 1(a) and 1(b), we assume a separable form for the tunnel coupling between the leads in Eq. (5). Here, we discuss the justification of this form using a tight-binding model. We also show that $\left|p_{\alpha}\right|<1$ in the presence of multiple conduction channels in lead $\alpha$.

As a simple example, let us consider the model depicted in Fig. 9(a). The leads consist of two sites in width and $N$ sites in length $(N \gg 1)$. The eigenvalues of the Hamiltonian for leads $L$ and $R$ form two subbands $\varepsilon_{ \pm}(q)$, where $q$ is the 


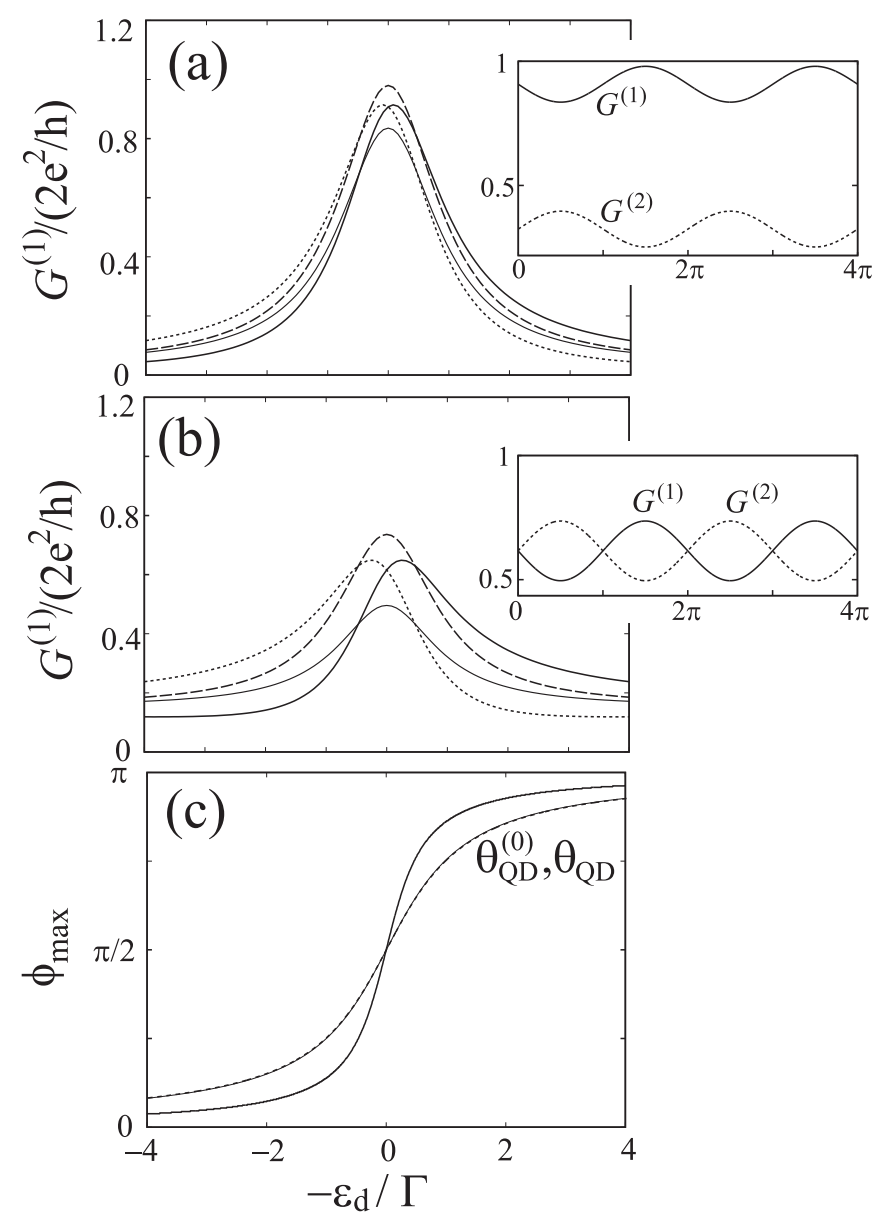

FIG. 7. Calculated results for the conductance and measured phase shift in the three-terminal model depicted in Fig. 4(b) in the absence of $U$. In the upper two panels, the conductance $G^{(1)}$ to lead $R(1)$ at temperature $T=0$ is plotted as a function of energy level $\varepsilon_{d}$ in the quantum dot. $\Gamma_{L}=\Gamma_{R}=\Gamma / 2, x=0.09\left(x_{L}=x_{R}=0.3\right)$, and $p_{L}=0.5$. (a) $\beta_{R}^{2}=0.1$, (b) $0.5\left(\alpha_{R}^{2}+\beta_{R}^{2}=1 ; \Gamma_{R}^{(1)}=\alpha_{R}^{2} \Gamma_{R}, \Gamma_{R}^{(2)}=\right.$ $\beta_{R}^{2} \Gamma_{R}, x_{R}^{(1)}=\beta_{R}^{2} x_{R}$, and $x_{R}^{(2)}=\alpha_{R}^{2} x_{R}$, see text). The $\mathrm{AB}$ phase for the magnetic flux penetrating the ring is $\phi=0$ (solid line), $\phi=\pi / 2$ (broken line), $\phi=\pi$ (dotted line), and $\phi=-\pi / 2$ (thin solid line). In panel (c), the measured phase shift $\phi_{\max }$ is plotted as a function of $\varepsilon_{d}$ (solid line), which is defined by the AB phase when $G^{(1)}(\phi)$ is maximal. $\phi_{\max }$ does not depend on $\beta_{R}$. The phase shift $\theta_{\mathrm{QD}}^{(0)}$ through the QD without the upper arm of the ring is plotted by dotted line, which is almost overlapped by $\theta_{\mathrm{QD}}$ (thin solid line) that satisfies the Friedel sum rule in the QD embedded in the ring. Insets in panels (a) and (b): $G^{(1)}$ and $G^{(2)}$ [conductance to lead $R(2)$ ] as a function of the $\mathrm{AB}$ phase $\phi$, at $\varepsilon_{d}=0$.

wave number in the $x$ direction $(0<q<\pi / a)$ with $a$ being the lattice constant [Fig. 9(b)]. The corresponding states are

$$
\begin{array}{r}
|L ; q, \pm\rangle=\frac{-1}{\sqrt{N+1}} \sum_{j=-N}^{-1}(|j, 1\rangle \pm|j, 2\rangle) \sin q j a \\
|R ; q, \pm\rangle=\frac{1}{\sqrt{N+1}} \sum_{j=1}^{N}(|j, 1\rangle \pm|j, 2\rangle) \sin q j a
\end{array}
$$

where $|j, \ell\rangle$ is the Wannier function at site $(j, \ell)$. The tunnel coupling between $|L ; q, \gamma\rangle$ and $\left|R ; q^{\prime}, \gamma^{\prime}\right\rangle\left(\gamma, \gamma^{\prime}= \pm\right)$
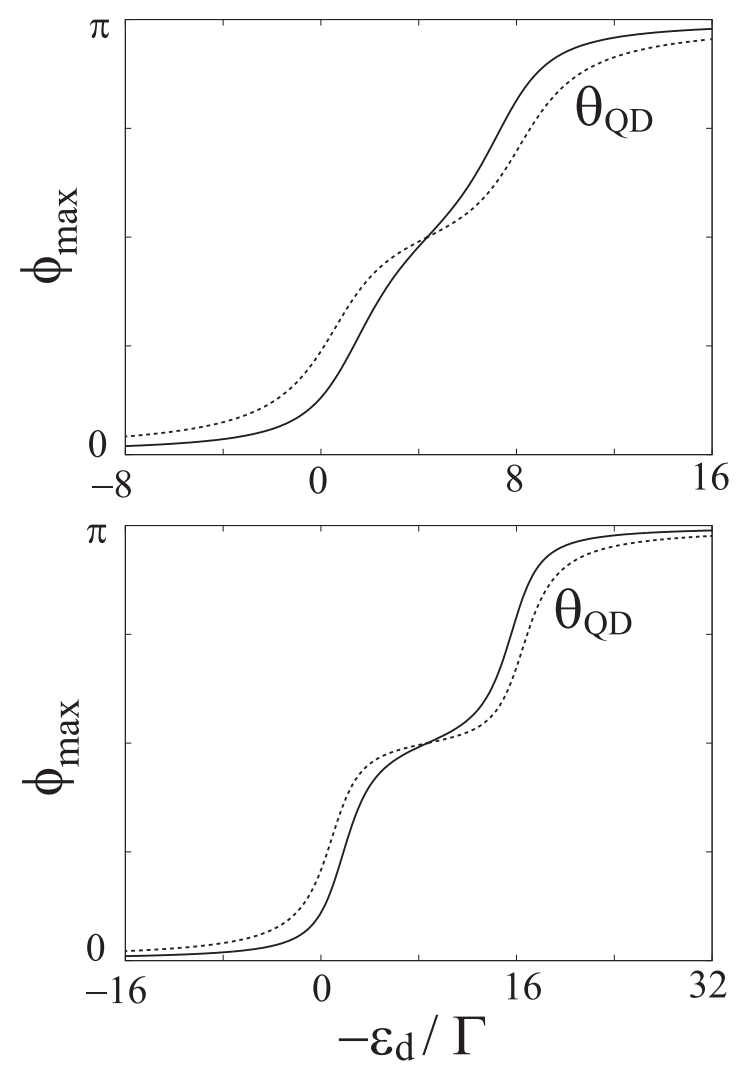

FIG. 8. Calculated results for the measured phase shift in the three-terminal model depicted in Fig. 4(b) in the presence of $U$. The measured phase shift $\phi_{\max }$ is plotted by solid line as a function of energy level $\varepsilon_{d}$ in the quantum dot. $\phi_{\max }$ is numerically evaluated as the $\mathrm{AB}$ phase at which the conductance $G^{(1)}(\phi)$ to lead $R(1)$ is maximal at temperature $T=0 . \Gamma_{L}=\Gamma_{R}=\Gamma / 2, x=0.09\left(x_{L}=x_{R}=0.3\right)$, and $p_{L}=0.5$. (a) $U / \Gamma=8$ and (b) 16. $\theta_{\mathrm{QD}}$ calculated from the Friedel sum rule, $\theta_{\mathrm{QD}}=\pi\left\langle n_{\sigma}\right\rangle$, is plotted by dotted line. $\phi_{\max }$ and $\theta_{\mathrm{QD}}$ are related to each other by Eq. (42).

is expressed as $W_{q^{\prime}, \gamma^{\prime} ; q, \gamma}=\psi_{R ; q^{\prime}, \gamma^{\prime}}(1,2) W \psi_{L ; q, \gamma}(-1,2)$ using the wave functions at the edge of the leads, $\psi_{L ; q, \pm}(-1,2)=$ $\langle-1,2 \mid L ; q, \pm\rangle$ and $\psi_{R ; q^{\prime}, \pm}(1,2)=\left\langle 1,2 \mid R ; q^{\prime}, \pm\right\rangle$. In consequence $W_{q^{\prime}, \gamma^{\prime} ; q, \gamma}$ has a separable form in Eq. (5) with

$$
\begin{aligned}
& \sqrt{w_{L ; q, \gamma}}=\sqrt{W} \psi_{L ; q, \gamma}(-1,2), \\
& \sqrt{w_{R ; q^{\prime}, \gamma^{\prime}}}=\sqrt{W} \psi_{R ; q^{\prime}, \gamma^{\prime}}(1,2) .
\end{aligned}
$$

When the Fermi level intersects both the subbands, there are two conduction channels, labeled by $k=(q, \pm)$, as indicated in Fig. 9(b). Then

$$
p_{L}=p_{R}=\frac{\sin q_{+} a-\sin q_{-} a}{\sin q_{+} a+\sin q_{-} a},
$$

where $q_{ \pm}$are the intersections between the subband \pm and Fermi level, as derived in Appendix D. Thus $\left|p_{L, R}\right|<1$. On the other hand, $p_{L, R}= \pm 1$, in the case of single conduction channel when $E_{\mathrm{F}}$ crosses one of the subbands.

Although we considered a specific model in Fig. 9(a), the separable form of $W_{k^{\prime}, k}$ in Eq. (5) should be justified when the system is described by a tight-binding model in general. Then $\sqrt{w_{L, k}}\left(\sqrt{w_{R, k^{\prime}}}\right)$ is proportional to the wave function 
(a)

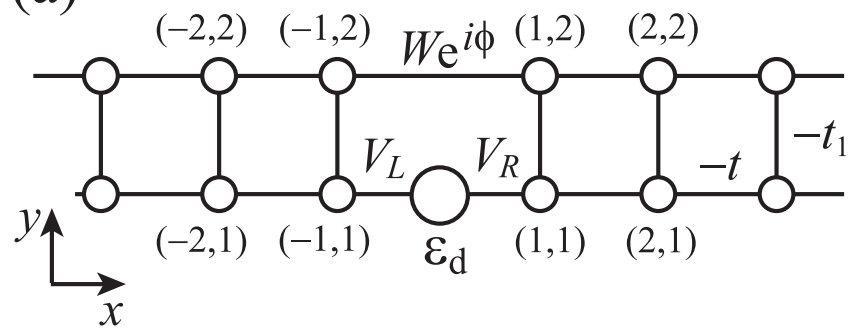

(b)

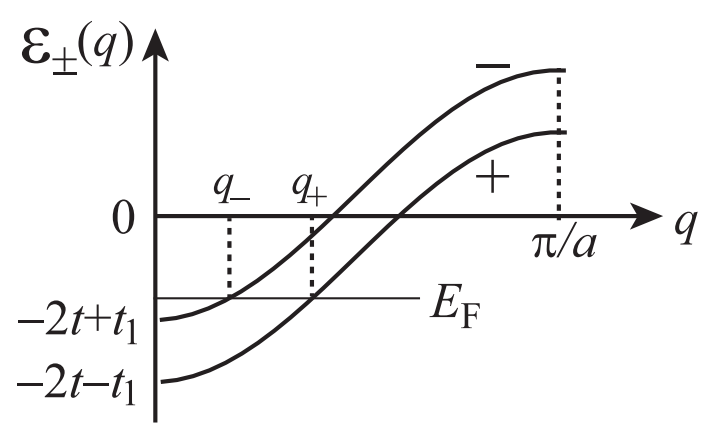

FIG. 9. (a) A tight-binding model for the QD interferometer. A QD is connected to sites $(-1,1)$ and $(1,1)$ by transfer integrals $V_{L}$ and $V_{R}$, respectively, whereas the upper arm of the ring couples sites $(-1,2)$ and $(1,2)$ by $W e^{ \pm i \phi}$ in $\pm x$ direction, where $\phi$ is the $\mathrm{AB}$ phase for the magnetic flux penetrating the ring (central rectangular region including the QD). Leads $L$ and $R$ consist of two sites in width ( $y$ direction) and $N$ sites in length ( $x$ direction; $N \gg 1$ ), in which the transfer integral is $-t\left(-t_{1}\right)$ in the $x(y)$ direction and the lattice constant is $a$. (b) Two subbands in the leads, $\varepsilon_{ \pm}(q)=\mp t_{1}-2 t \cos q a$, as a function of wave number $q$ in the $x$ direction $(0<q<\pi / a)$. There are two conduction channels when the Fermi level $E_{\mathrm{F}}$ intersects both the subbands at $q=q_{ \pm}$.

$\psi_{L, k}\left(\psi_{R, k^{\prime}}\right)$ at the edge of the lead, as in Eqs. (45) and (46). We could also claim that $p_{L, R}<1$ for the leads of multiple conduction channels and $p_{L, R}=1$ for the leads of single channel in the usual cases. Precisely speaking, the presence of multiple channels is a necessary condition for $p_{L, R}<1: p_{\alpha}$ is determined by the detailed shape of the system around a junction between the ring and lead $\alpha$ through Eq. (10).

We comment on the generality of our models. In this section, we examined a model in which the subbands $( \pm)$ are well defined in the leads. Then the state in the leads is labeled by $k=(q, \pm)$ in the presence of two conduction channels. This is not the case in experimental systems of various shape. We believe that $\Gamma_{\alpha}, x_{\alpha}$, and $p_{\alpha}$ can be defined in Eqs. (6) to (8) using state-dependent tunnel couplings without loss of generality. In our models in Figs. 1(a) and 1(b), we assume a single conduction channel in the upper arm of the ring. The multiple channels in the arm should be beyond the scope of our study.

\section{CONCLUSION}

We theoretically examined the transport through an Aharonov-Bohm ring with an embedded quantum dot (QD), the so-called QD interferometer, to address two controversial issues, one concerns the shape of the conductance peak as a function of energy level $\varepsilon_{d}$ in the QD and the other is about the phase measurement in the multiterminal geometry as a double-slit experiment. For this purpose, we generalized a previous model in Refs. $[18,19]$ to consider multiple conduction channels in leads $L$ and $R$. In our model, the tunnel couplings between the QD and leads and that between the leads depend on the states in the leads, as shown in Figs. 1(a) and 1(b). This gives rise to a parameter $p_{\alpha}\left(\left|p_{\alpha}\right| \leqslant 1\right)$ to characterize a connection between the two arms of the ring through lead $\alpha$ $(=L, R)$, which is equal to the overlap integral between the conduction modes coupled to the upper and lower arms of the ring.

First, we examined the shape of the conductance peak in the two-terminal geometry, in the absence of electron-electron interaction $U$ in the QD. We showed an asymmetric Fano resonance at $\left|p_{L, R}\right| \approx 1$ and an almost symmetric Breit-Wigner resonance at $\left|p_{L, R}\right|<0.5$. Hence our model could explain the experimental results of both an asymmetric Fano resonance [5] and almost symmetric Breit-Wigner resonance [8], with fitting parameters $p_{L, R}$ to their data.

Second, we took into account the Kondo effect in the presence of $U$, using the Bethe ansatz exact solution, and precisely evaluated the conductance at temperature $T=0$. We showed a crossover from an asymmetric Fano-Kondo resonance [19] to the Kondo plateau with changing $p_{L, R}$.

Our model is also applicable to the multiterminal geometry to address the second issue on the measurement of the transmission phase shift through the QD by a double-slit experiment. We studied the measured phase $\phi_{\max }$, the AB phase at which the conductance $G^{(1)}(\phi)$ to lead $R(1)$ is maximal in Fig. 1(b). In the absence of $U$, Eq. (37) indicates the relation of $\phi_{\max }$ to an intrinsic phase shift $\theta_{\mathrm{QD}}^{(0)}$ that is the phase shift through the QD without the upper arm of the ring. We examined two specific models in the three-terminal geometry, depicted in Fig. 4. We discussed a crossover from two- to three-terminal phase measurement in the former and simulated the experimental system consisting of two quantum wires $[8,16,17]$ in the latter. Using the latter model, we showed how precisely the phase locking at $\pi / 2$ is measured in the Kondo regime.

\section{ACKNOWLEDGMENTS}

We appreciate fruitful discussions with Dr. Akira Oguri. This work was partially supported by JSPS KAKENHI Grants No. JP26220711 and No. JP15H05870 and JST-CREST Grant No. JPMJCR1876.

\section{APPENDIX A: CURRENT FORMULATION USING KELDYSH GREEN'S FUNCTIONS}

The current is formulated for the multiterminal model depicted in Fig. 1(b), using the Keldysh Green's functions [43-45]. The chemical potential in lead $L(j)[R(j)]$ is denoted by $\mu_{L}^{(j)}\left[\mu_{R}^{(j)}\right]$. The spin index $\sigma$ is omitted in this Appendix. 


\section{Keldysh Green's functions}

The retarded, advanced, and lesser Green's functions are defined by

$$
\begin{aligned}
& G_{d, L k}^{\mathrm{r}}\left(t, t^{\prime}\right)=\frac{1}{i \hbar}\left\langle\left\{d(t), a_{L k}^{\dagger}\left(t^{\prime}\right)\right\}\right\rangle \theta\left(t-t^{\prime}\right), \\
& G_{d, L k}^{\mathrm{a}}\left(t, t^{\prime}\right)=-\frac{1}{i \hbar}\left\langle\left\{d(t), a_{L k}^{\dagger}\left(t^{\prime}\right)\right\}\right\rangle \theta\left(t^{\prime}-t\right), \\
& G_{d, L k}^{<}\left(t, t^{\prime}\right)=\frac{-1}{i \hbar}\left\langle a_{L k}^{\dagger}\left(t^{\prime}\right) d(t)\right\rangle,
\end{aligned}
$$

respectively, where $\{A, B\}=A B+B A$ and $\theta(t)$ is the Heaviside step function. The other Green's functions, $G_{d, d}^{\lambda}$, $G_{L k, R k^{\prime}}^{\lambda}$, and so on $(\lambda=\mathrm{r}, \mathrm{a},<)$, are defined in a similar manner. The average is taken for the stationary state and hence all the Green's functions depend on $t-t^{\prime}$ only. Note that $G_{d, L k}^{<}\left(t-t^{\prime}\right)=-\left[G_{L k, d}^{<}\left(t^{\prime}-t\right)\right]^{*}$ and $G_{d, d}^{<}\left(t-t^{\prime}\right)=-\left[G_{d, d}^{<}\left(t^{\prime}-t\right)\right]^{*}$. The Fourier transformation $\left(t-t^{\prime} \rightarrow \omega\right)$ yields $G_{d, L k}^{<}(\omega)=-\left[G_{L k, d}^{<}(\omega)\right]^{*}$ and $G_{d, d}^{<}(\omega)=$ $-\left[G_{d, d}^{<}(\omega)\right]^{*}$.

We also introduce the Green's functions in isolate leads $L$ and $R$, in the absence of tunnel coupling, $H_{T}$ in Eq. (4). For example,

$$
\begin{aligned}
g_{L k}^{\mathrm{r}}\left(t, t^{\prime}\right) & =\frac{1}{i \hbar}\left\langle\left\{a_{L k}(t), a_{L k}^{\dagger}\left(t^{\prime}\right)\right\}\right\rangle \theta\left(t-t^{\prime}\right) \\
& =\frac{1}{i \hbar} e^{-i \varepsilon_{k}\left(t-t^{\prime}\right) / \hbar} \theta\left(t-t^{\prime}\right),
\end{aligned}
$$

$$
\begin{aligned}
g_{L k}^{<}\left(t, t^{\prime}\right) & =\frac{-1}{i \hbar}\left\langle a_{L k}^{\dagger}\left(t^{\prime}\right) a_{L k}(t)\right\rangle \\
& =\frac{-1}{i \hbar} f_{L}^{(j)}\left(\varepsilon_{k}\right) e^{-i \varepsilon_{k}\left(t-t^{\prime}\right) / \hbar},
\end{aligned}
$$

where $f_{L}^{(j)}(\varepsilon)=\left[\left(\varepsilon-\mu_{L}^{(j)}\right) /\left(k_{\mathrm{B}} T\right)+1\right]^{-1}$ is the Fermi distribution function in lead $L(j)$ that state $k$ belongs to ( $j=1$ or 2). The Fourier transformation leads to

$$
\begin{aligned}
g_{L k}^{\mathrm{r}}(\omega) & =\frac{1}{\hbar \omega-\varepsilon_{k}+i \delta} \\
& =P \frac{1}{\hbar \omega-\varepsilon_{k}}-i \pi \delta\left(\hbar \omega-\varepsilon_{k}\right), \\
g_{L k}^{<}(\omega) & =2 \pi i f_{L}^{(j)}(\hbar \omega) \delta\left(\hbar \omega-\varepsilon_{k}\right) .
\end{aligned}
$$

In the following calculations, the real part (principal value) of $g_{\alpha k}^{\mathrm{r}}(\omega)$ and $g_{\alpha k}^{\mathrm{a}}(\omega)=\left[g_{\alpha k}^{\mathrm{r}}(\omega)\right]^{*}$ is disregarded in the summation over $k$, assuming a wide band limit.

In the next subsection, $G_{d, L k}^{<}$is replaced by $G_{d, d}^{\mathrm{r}}$ and $G_{d, d}^{<}$. For this purpose, their relation is derived in the following. In the Baym-Kadanoff-Keldysh nonequilibrium techniques, a complex-time contour is considered from $t=-\infty$ to $t=t_{0}$ just above the real axis and from $t=t_{0}$ to $t=-\infty$ just below the real axis. For the contour-ordered Green's function,

$$
G_{d, L k}^{\mathrm{C}}\left(\tau, \tau^{\prime}\right)=\frac{1}{i \hbar}\left\langle\mathcal{T}_{\mathrm{C}} d(\tau) a_{L k}^{\dagger}\left(\tau^{\prime}\right)\right\rangle
$$

the equation-of-motion method yields $[44,45]$

$$
G_{d, L k}^{\mathrm{C}}\left(\tau, \tau^{\prime}\right)=\int d \tau_{1}\left[G_{d, d}^{\mathrm{C}}\left(\tau, \tau_{1}\right) V_{L k}+\sum_{k^{\prime}}^{(1),(2)} G_{d, R k^{\prime}}^{\mathrm{C}}\left(\tau, \tau_{1}\right) W_{k^{\prime}, k} e^{i \phi}\right] g_{L k}^{\mathrm{C}}\left(\tau_{1}, \tau^{\prime}\right) .
$$

According to the Langreth's theorem $[45,46]$, this results in

$$
G_{d, L k}^{\mathrm{r}}\left(t, t^{\prime}\right)=\int d t_{1}\left[G_{d, d}^{\mathrm{r}}\left(t, t_{1}\right) V_{L k}+\sum_{k^{\prime}}^{(1),(2)} G_{d, R k^{\prime}}^{\mathrm{r}}\left(t, t_{1}\right) W_{k^{\prime}, k} e^{i \phi}\right] g_{L k}^{\mathrm{r}}\left(t_{1}, t^{\prime}\right),
$$

and

$$
\begin{aligned}
G_{d, L k}^{<}\left(t, t^{\prime}\right)=\int d t_{1}\{ & {\left[G_{d, d}^{\mathrm{r}}\left(t, t_{1}\right) V_{L k}+\sum_{k^{\prime}}^{(1),(2)} G_{d, R k^{\prime}}^{\mathrm{r}}\left(t, t_{1}\right) W_{k^{\prime}, k} e^{i \phi}\right] g_{L k}^{<}\left(t_{1}, t^{\prime}\right) } \\
+ & {\left.\left[G_{d, d}^{<}\left(t, t_{1}\right) V_{L k}+\sum_{k^{\prime}}^{(1),(2)} G_{d, R k^{\prime}}^{<}\left(t, t_{1}\right) W_{k^{\prime}, k} e^{i \phi}\right] g_{L k}^{\mathrm{a}}\left(t_{1}, t^{\prime}\right)\right\} . }
\end{aligned}
$$

Similar relations are obtained for $G_{d, R k^{\prime}}^{\mathrm{r}}$, and so on.

\section{Current formula using $G_{d, d}^{\mathrm{r}}$ and $G_{d, d}^{<}$}

We express the current from lead $L(1)$ in terms of $G_{d, d}^{\mathrm{r}}$ and $G_{d, d}^{<}$. The substitution of the Hamiltonian in Eq. (1) into Eq. (15) results in

$$
I_{L}^{(1)}=-\frac{2 e}{i \hbar} \sum_{k}^{(1)}\left[V_{L k}\left\langle a_{L k}^{\dagger} d-d^{\dagger} a_{L k}\right\rangle+\sum_{k^{\prime}}^{(1),(2)} W_{k^{\prime}, k}\left\langle e^{-i \phi} a_{L k}^{\dagger} a_{R k^{\prime}}-e^{i \phi} a_{R k^{\prime}}^{\dagger} a_{L k}\right\rangle\right] .
$$


We added a factor of 2 by the summation over spin index $\sigma$. This equation is rewritten as

$$
\begin{aligned}
I_{L}^{(1)} & =4 e \operatorname{Re} \sum_{k}^{(1)}\left[V_{L k} G_{d, L k}^{<}(t, t)+\sum_{k^{\prime}}^{(1),(2)} W_{k^{\prime}, k} e^{-i \phi} G_{R k^{\prime}, L k}^{<}(t, t)\right] \\
& =\frac{4 e}{2 \pi} \operatorname{Re} \int d \omega \sum_{k}^{(1)}\left[V_{L k} G_{d, L k}^{<}(\omega)+\sum_{k^{\prime}}^{(1),(2)} W_{k^{\prime}, k} e^{-i \phi} G_{R k^{\prime}, L k}^{<}(\omega)\right] .
\end{aligned}
$$

Hence we need to calculate two terms in the integral,

$$
\begin{aligned}
X_{0} & =\sum_{k}^{(1)} V_{L k} G_{d, L k}^{<}(\omega) \\
Y_{0} & =\sum_{k}^{(1)} \sum_{k^{\prime}}^{(1),(2)} W_{k^{\prime}, k} e^{-i \phi} G_{R k^{\prime}, L k}^{<}(\omega) .
\end{aligned}
$$

Let us consider $X_{0}$. Using the Fourier transformation of Eq. (A11), we obtain

$$
\begin{aligned}
X_{0}= & i \Gamma_{L}^{(1)}\left[2 f_{L}^{(1)}(\hbar \omega) G_{d, d}^{\mathrm{r}}(\omega)+G_{d, d}^{<}(\omega)\right] \\
& +i \tilde{p}_{L}^{(1)} e^{i \phi} \sum_{k^{\prime}}^{(1),(2)} \sqrt{w_{R k^{\prime}}}\left[2 f_{L}^{(1)}(\hbar \omega) G_{d, R k^{\prime}}^{\mathrm{r}}(\omega)+G_{d, R k^{\prime}}^{<}(\omega)\right],
\end{aligned}
$$

where $\tilde{p}_{\alpha}^{(j)}=\sqrt{\Gamma_{\alpha}^{(j)} x_{\alpha}^{(j)}} p_{\alpha}^{(j)}$. Then we need

$$
\begin{aligned}
& X_{1}=\sum_{k^{\prime}}^{(1),(2)} \sqrt{w_{R k^{\prime}}} G_{d, R k^{\prime}}^{\mathrm{r}}(\omega), \\
& X_{2}=\sum_{k^{\prime}}^{(1),(2)} \sqrt{w_{R k^{\prime}}} G_{d, R k^{\prime}}^{<}(\omega) .
\end{aligned}
$$

For $X_{1}$, we use an equation for $G_{d, R k^{\prime}}^{\mathrm{r}}$ corresponding to Eq. (A10) for $G_{d, L k}^{\mathrm{r}}$, which leads to

$$
X_{1}=-i\left[\tilde{p}_{R}^{(1)}+\tilde{p}_{R}^{(2)}\right] G_{d, d}^{\mathrm{r}}(\omega)-i x_{R} e^{-i \phi} Y_{1}
$$

with

$$
Y_{1}=\sum_{k}^{(1),(2)} \sqrt{w_{L k}} G_{d, L k}^{\mathrm{r}}(\omega)
$$

Using the Fourier transformation of Eq. (A10), we obtain

$$
Y_{1}=-i\left[\tilde{p}_{L}^{(1)}+\tilde{p}_{L}^{(2)}\right] G_{d, d}^{\mathrm{r}}(\omega)-i x_{L} e^{i \phi} X_{1}
$$

From Eqs. (A19) and (A21), we express $X_{1}$ in terms of $G_{d, d}^{\mathrm{r}}(\omega)$. In the same way, $X_{2}$ can be written using $G_{d, d}^{\mathrm{r}}(\omega)$ and $G_{d, d}^{<,}(\omega)$.

A similar procedure is adopted for $Y_{0}$. The final result is so lengthy that we show the current expression in the case of Eq. (17), i.e., $\mu_{L}^{(1)}=\mu_{L}^{(2)} \equiv \mu_{L}$ and $\mu_{R}^{(1)}=\mu_{R}^{(2)} \equiv \mu_{R}$. After the variable conversion of $\hbar \omega \rightarrow \varepsilon$,

$$
\begin{aligned}
I_{L}^{(1)}= & \frac{4 e}{h} \int d \varepsilon\left\{-\Gamma_{L}^{(1)}\left[2 f_{L}(\varepsilon) \operatorname{Im} G_{d, d}^{\mathrm{r}}(\varepsilon)+\operatorname{Im} G_{d, d}^{<}(\varepsilon)\right]+x_{L}^{(1)} x_{R} \frac{2}{(1+x)^{2}}\left[f_{L}(\varepsilon)-f_{R}(\varepsilon)\right]\right. \\
& \left.+\tilde{p}_{L}^{(1)}\left[A_{1} \operatorname{Re} G_{d, d}^{\mathrm{r}}(\varepsilon)+A_{2} \operatorname{Im} G_{d, d}^{\mathrm{r}}(\varepsilon)+A_{3} \operatorname{Im} G_{d, d}^{<}(\varepsilon)\right]+x_{L}^{(1)}\left[B_{1} \operatorname{Re} G_{d, d}^{\mathrm{r}}(\varepsilon)+B_{2} \operatorname{Im} G_{d, d}^{\mathrm{r}}(\varepsilon)+B_{3} \operatorname{Im} G_{d, d}^{<}(\varepsilon)\right]\right\}
\end{aligned}
$$

where

$$
\begin{aligned}
& A_{1}=\frac{4}{(1+x)^{2}} \tilde{p}_{R} \cos \phi\left[f_{L}(\varepsilon)-f_{R}(\varepsilon)\right], \\
& A_{2}=\frac{4}{(1+x)^{2}}\left\{f_{L}(\varepsilon)\left[x \tilde{p}_{R} \sin \phi+(2+x) x_{R} \tilde{p}_{L}\right]+f_{R}(\varepsilon)\left[\tilde{p}_{R} \sin \phi-x_{R} \tilde{p}_{L}\right]\right\} \\
& A_{3}=\frac{2}{1+x}\left(\tilde{p}_{R} \sin \phi+x_{R} \tilde{p}_{L}\right), \\
& B_{1}=-\frac{8}{(1+x)^{3}} x_{R} \tilde{p}_{L} \tilde{p}_{R} \cos \phi\left[f_{L}(\varepsilon)-f_{R}(\varepsilon)\right], \\
& B_{2}=\frac{2}{(1+x)^{3}}\left\{f_{L}(\varepsilon)\left[-2(1+x) x_{R} \tilde{p}_{L} \tilde{p}_{R} \sin \phi+(1-x) \tilde{p}_{R}^{2}-(3+x) x_{R}^{2} \tilde{p}_{L}^{2}\right]-2 f_{R}(\varepsilon)\left(\tilde{p}_{R}^{2}-x_{R}^{2} \tilde{p}_{L}^{2}\right)\right\}, \\
& B_{3}=-\frac{1}{(1+x)^{2}}\left(2 x_{R} \tilde{p}_{L} \tilde{p}_{R} \sin \phi+\tilde{p}_{R}^{2}+x_{R}^{2} \tilde{p}_{L}^{2}\right),
\end{aligned}
$$

with $\tilde{p}_{\alpha}=\tilde{p}_{\alpha}^{(1)}+\tilde{p}_{\alpha}^{(2)}=\sqrt{\Gamma_{\alpha} x_{\alpha}} p_{\alpha}$.

The current $I_{L}^{(2)}$ from lead $L(2)$ is given by replacing $(1) \rightarrow(2)$ in Eq. (A22). The current $I_{R}^{(j)}$ from lead $R(j)$ is obtained from $I_{L}^{(j)}$ by replacing $L \leftrightarrow R$ and $\phi \rightarrow-\phi$. These equations yield Eq. (18) for the current conservation. 


\section{Current formula in terms of $G_{d, d}^{\mathrm{r}}$}

For the two-terminal model in Fig. 1(a), the current from lead $L$ is $I_{L}=I_{L}^{(1)}+I_{L}^{(2)}$. The elimination of $G_{d, d}^{<}$using Eq. (18) results in its expression in Eq. (20).

As a three-terminal model, we examine the model in Fig. 1(b) consisting of leads $L, R(1)$, and $R(2)$. We introduce parameters, $\gamma_{R}^{(j)}, y_{R}^{(j)}$, and $q_{R}^{(j)}$ in Eq. (34). The current into lead $R(1)$ is given by $-I_{R}^{(1)}$. Eliminating $G_{d, d}^{<}$using Eq. (18), we obtain

$$
\begin{gathered}
-I_{R}^{(1)}=\frac{2 e}{h} \int d \varepsilon\left[f_{L}(\varepsilon)-f_{R}(\varepsilon)\right] T^{(1)}(\varepsilon) d \varepsilon, \\
T^{(1)}(\varepsilon)=\frac{4 x}{(1+x)^{2}} y_{R}^{(1)}+8 \frac{(1+x) q_{R}^{(1)}-2 x y_{R}^{(1)}}{(1+x)^{3}} \sqrt{\Gamma_{L} \Gamma_{R} x} p_{L} p_{R} \cos \phi \operatorname{Re} G_{d, d}^{\mathrm{r}}(\varepsilon)+\frac{4 C_{2}}{(1+x)^{3} \tilde{\Gamma}} \operatorname{Im} G_{d, d}^{\mathrm{r}}(\varepsilon),
\end{gathered}
$$

where

$$
\begin{aligned}
C_{2}= & -2(1+x) \sqrt{\Gamma_{L} \Gamma_{R} x} p_{L} p_{R} \sin \phi\left[x\left(q_{R}^{(1)}-y_{R}^{(1)}\right) \Gamma_{L}\left(1-p_{L}^{2}\right)+\left(\gamma_{R}^{(1)}-q_{R}^{(1)}\right) \Gamma_{R}\right]+\frac{x^{3}}{1+x} y_{R}^{(1)}\left[\left(\Gamma_{L} p_{L}^{2}\right)^{2}+\left(\Gamma_{R} p_{R}^{2}\right)^{2}\right] \\
& +x(1-x) y_{R}^{(1)}\left(\Gamma_{L} p_{L}\right)^{2}+x\left[(1+x)\left(-\gamma_{R}^{(1)}+2 q_{R}^{(1)}\right)-2 x y_{R}^{(1)}\right]\left(\Gamma_{R} p_{R}\right)^{2}-\Gamma_{L} \Gamma_{R} D_{2},
\end{aligned}
$$

with

$$
\begin{aligned}
D_{2}= & (1+x)^{3} \gamma_{R}^{(1)}+4 x \frac{(1+x) q_{R}^{(1)}-x y_{R}^{(1)}}{1+x}\left(p_{L} p_{R}\right)^{2} \sin ^{2} \phi+\frac{x^{2}\left[2(x+3)(x+1) q_{R}^{(1)}-\left(x^{2}+4 x-3\right) y_{R}^{(1)}\right]}{1+x}\left(p_{L} p_{R}\right)^{2} \\
& -x\left[(x+1)(x+2) \gamma_{R}^{(1)}+2 y_{R}^{(1)}\right] p_{L}^{2}-x\left[2(x+1)(x+2) q_{R}^{(1)}-x(x+3) y_{R}^{(1)}\right] p_{R}^{2} .
\end{aligned}
$$

Regarding the $\phi$-dependence of the conductance at $T=0$, Eqs. (A29) and (A30) yield Eqs. (35) and (36) in the absence of $U$. In the presence of $U$, however, we cannot obtain such a simple form in general.

\section{APPENDIX B: GREEN'S FUNCTION IN THE PRESENCE OF $U$}

For our models shown in Figs. 1(a) and 1(b), the Green's function of the QD is solvable in the case of $U=0$. As discussed in Sec. II.D, the retarded Green's function is given by

$$
G_{d, d}^{\mathrm{r}}(\varepsilon)=\frac{1}{\varepsilon-\tilde{\varepsilon}_{d}(\phi)+i \tilde{\Gamma}}
$$

with the effective energy level $\tilde{\varepsilon}_{d}(\phi)$ in Eq. (25) and effective linewidth $\tilde{\Gamma}$ in Eq. (24). The renormalization due to the direct tunneling between the leads and the Aharonov-Bohm effect by the magnetic flux is included in these effective parameters.

In the presence of $U$, we formulate the perturbation with respect to the electron-electron interaction in the QD, $H_{U}=U n_{\uparrow} n_{\downarrow}$. The Hamiltonian in Eq. (1) is divided into the noninteracting part $H_{0}$ and $H_{U} ; H=H_{0}+H_{U}$. The contour-ordered Green's function of the $\mathrm{QD}, G_{d, d}^{\mathrm{C}}\left(\tau, \tau^{\prime}\right)=$ $\left\langle\mathcal{T}_{\mathrm{C}} d_{\sigma}(\tau) d_{\sigma}^{\dagger}\left(\tau^{\prime}\right)\right\rangle /(i \hbar)$, is written as

$$
\begin{aligned}
& G_{d, d}^{\mathrm{C}}\left(\tau, \tau^{\prime}\right) \\
& \quad=\frac{1}{i \hbar} \operatorname{tr}\left\{\rho_{0} \mathcal{T}_{\mathrm{C}} d_{\mathrm{I}, \sigma}(\tau) d_{\mathrm{I}, \sigma}^{\dagger}\left(\tau^{\prime}\right) \exp \left[\int_{\mathrm{C}} d \tau^{\prime \prime} H_{\mathrm{I}, U}\left(\tau^{\prime \prime}\right)\right]\right\},
\end{aligned}
$$

where $\rho_{0}$ is the density matrix for $U=0$ and index I indicates the operator in the interaction picture, $\mathcal{O}_{\mathrm{I}}(\tau)=$ $e^{i H_{0} \tau / \hbar} \mathcal{O} e^{-i H_{0} \tau / \hbar}$. In the perturbative expansion, the unperturbed Green's function is given by Eq. (B1). This problem is equivalent to that of the conventional Anderson impu- rity model, in which an impurity with energy level $\tilde{\varepsilon}_{d}(\phi)$ and Coulomb interaction $U$ is connected to an energyband of conduction electrons via the effective hybridization $\tilde{\Gamma}$ :

$$
\begin{aligned}
H_{\text {Anderson }}= & \tilde{\varepsilon}_{d}(\phi) \sum_{\sigma} n_{\sigma}+U n_{\uparrow} n_{\downarrow}+\sum_{k \sigma} \varepsilon_{k} a_{k, \sigma}^{\dagger} a_{k, \sigma} \\
& +\sum_{k \sigma}\left(v a_{k, \sigma}^{\dagger} d_{\sigma}+\text { H.c. }\right),
\end{aligned}
$$

where $\tilde{\Gamma}=\pi \rho|v|^{2}$, with the density of states $\rho$ for the conduction electrons.

In the equilibrium with $e V=0$, the physical quantities of electrons in our model can be evaluated by exploiting the established methods for the Anderson impurity model [19]. The retarded Green's function is given by

$$
G_{d, d}^{\mathrm{r}}(\varepsilon)=\frac{1}{\varepsilon-\tilde{\varepsilon}_{d}(\phi)+i \tilde{\Gamma}-\Sigma_{U}(\varepsilon)}
$$

with use of the self-energy $\Sigma_{U}(\varepsilon)$ due to the electron-electron interaction in the QD. Note that $z=\left[1-\frac{d \Sigma_{U}}{d \varepsilon}(0)\right]^{-1}$ and $\tilde{\varepsilon}_{d}^{*}=$ $z\left[\tilde{\varepsilon}_{d}(\phi)+\Sigma_{U}(0)\right]$ in Eq. (26). $G_{d, d}^{\mathrm{r}}(0)$ is expressed in Eq. (27) using the phase shift $\theta_{\mathrm{QD}}$. The Friedel sum rule connects the phase shift to the electron occupation per spin in the QD, $\theta_{\mathrm{QD}}=\pi\left\langle n_{\sigma}\right\rangle$, where

$$
\left\langle n_{\sigma}\right\rangle=\frac{1}{2}-\frac{1}{\pi} \tan ^{-1}\left(\frac{\tilde{\varepsilon}_{d}(\phi)+\Sigma_{U}(0)}{\tilde{\Gamma}}\right) .
$$

We use the Bethe ansatz exact solution to evaluate $\left\langle n_{\sigma}\right\rangle$ $[39,40]$.

\section{APPENDIX C: CURRENT IN THREE-TERMINAL MODEL IN FIG. 4(b)}

We apply the current formula in Eqs. (A29) and (A30) to the model in Fig. 4(b). 
As mentioned in Sec. IV C, $V_{R, k^{\prime}}=V_{R} \alpha_{R}$ and $\sqrt{w_{R, k^{\prime}}}=$ $\sqrt{w_{R}} \beta_{R}$ when state $k^{\prime}$ belongs to lead $R(1)$ while $V_{R, k^{\prime}}=V_{R} \beta_{R}$ and $\sqrt{w_{R, k^{\prime}}}=-\sqrt{w_{R}} \alpha_{R}$ when state $k^{\prime}$ belongs to lead $R(2)$ in the tunnel Hamiltonian $H_{T}$. This results in $\Gamma_{R}^{(1)}=\alpha_{R}^{2} \Gamma_{R}$, $\Gamma_{R}^{(2)}=\beta_{R}^{2} \Gamma_{R}, x_{R}^{(1)}=\beta_{R}^{2} x_{R}$, and $x_{R}^{(2)}=\alpha_{R}^{2} x_{R}$. We also find that $p_{R}^{(1)}=1, p_{R}^{(2)}=-1$, and hence $p_{R}=p_{R}^{(1)}+p_{R}^{(2)}=0$.

From $p_{R}=0, \tilde{\varepsilon}_{d}(\phi)=\varepsilon_{d}$ in Eq. (25), which is independent of the AB phase $\phi$ for the magnetic flux. The Green's function in the absence of $U$ becomes

$$
G_{d, d}^{\mathrm{r}}(\varepsilon)=\frac{1}{\varepsilon-\varepsilon_{d}+i \tilde{\Gamma}}
$$

with

$$
\tilde{\Gamma}=\Gamma_{L}\left(1-\frac{x}{1+x} p_{L}^{2}\right)+\Gamma_{R} .
$$

The substitution of $\gamma_{R}^{(1)}=\alpha_{R}^{2}, y_{R}^{(1)}=\beta_{R}^{2}$, and $q_{R}^{(1)} p_{R}=\alpha_{R} \beta_{R}$ $\left(q_{R}^{(1)}=\infty\right)$ into Eq. (A30) results in

$$
\begin{aligned}
T^{(1)}(\varepsilon)= & \frac{4 x}{(1+x)^{2}} \beta_{R}^{2}+\frac{8 \alpha_{R} \beta_{R}}{(1+x)^{2}} \sqrt{\Gamma_{L} \Gamma_{R} x} p_{L} \cos \phi \operatorname{Re} G_{d, d}^{\mathrm{r}}(\varepsilon) \\
& +\frac{4 C_{2}^{\prime}}{(1+x)^{3} \tilde{\Gamma}} \operatorname{Im} G_{d, d}^{\mathrm{r}}(\varepsilon),
\end{aligned}
$$

where

$$
\begin{aligned}
C_{2}^{\prime}= & -2(1+x) \sqrt{\Gamma_{L} \Gamma_{R} x} p_{L} \sin \phi\left[x \Gamma_{L}\left(1-p_{L}^{2}\right)-\Gamma_{R}\right] \alpha_{R} \beta_{R} \\
& +\frac{x^{3}}{1+x} \beta_{R}^{2}\left(\Gamma_{L} p_{L}^{2}\right)^{2}+x(1-x) \beta_{R}^{2}\left(\Gamma_{L} p_{L}\right)^{2} \\
& -\Gamma_{L} \Gamma_{R}\left\{(1+x)^{3} \alpha_{R}^{2}-x\left[(x+1)(x+2) \alpha_{R}^{2}+2 \beta_{R}^{2}\right] p_{L}^{2}\right\} .
\end{aligned}
$$

Since $\tilde{\varepsilon}_{d}(\phi)=\varepsilon_{d}$ in this model, Eq. (37) exactly holds in the absence of $U$, which leads to Eq. (41). In addition, even in the presence of $U$, a relation between $\phi_{\max }$ and $\theta_{\mathrm{QD}}$ is derived in the following. The substitution of Eq. (26) into Eq. (C3) yields

$$
\begin{aligned}
T^{(1)}(0)= & \frac{8 \alpha_{R} \beta_{R}}{(1+x)^{2}} \sqrt{\Gamma_{L} \Gamma_{R} x} p_{L} \frac{\tilde{\Gamma}^{*}}{\tilde{\Gamma}} \frac{1}{\left(\tilde{\varepsilon}_{d}^{*}\right)^{2}+\left(\tilde{\Gamma}^{*}\right)^{2}} F_{1}(\phi) \\
& +(\phi \text {-indep. terms })
\end{aligned}
$$

at $\varepsilon=E_{\mathrm{F}}=0$, where

$$
F_{1}(\phi)=-\tilde{\varepsilon}_{d}^{*} \cos \phi+\left[x \Gamma_{L}\left(1-p_{L}^{2}\right)-\Gamma_{R}\right] \frac{\tilde{\Gamma}^{*}}{\tilde{\Gamma}} \sin \phi .
$$

For $\phi=\phi_{\max }$ at which $F_{1}(\phi)$ is maximal,

$$
\tan \phi_{\max }=\frac{-x \Gamma_{L}\left(1-p_{L}^{2}\right)+\Gamma_{R}}{\tilde{\Gamma}} \tan \theta_{\mathrm{QD}},
$$

where $\tan \theta_{\mathrm{QD}}=\tilde{\Gamma}^{*} / \tilde{\varepsilon}_{d}^{*} . \theta_{\mathrm{QD}}$ satisfies the Friedel sum rule in the presence of $U$.

The current to lead $R(2),-I_{R}^{(2)}$, is given by replacing $(1) \rightarrow(2)$ in Eq. (A29). $T_{R}^{(2)}$ is obtained from $T_{R}^{(1)}$ in Eq. (C3), replacing $\alpha_{R} \rightarrow \beta_{R}$ and $\beta_{R} \rightarrow-\alpha_{R}$. In $T_{R}^{(1)}$ and $T_{R}^{(2)}$, coefficients of $\cos \phi$ and $\sin \phi$ are the same in magnitude and opposite in sign. As a result, the total current to leads $R(1)$ and $R(2)$ does not depend on the $\mathrm{AB}$ phase $\phi$ for the magnetic flux:

$$
-I_{R}^{(1)}-I_{R}^{(2)}=\frac{2 e}{h} \int\left[f_{L}(\varepsilon)-f_{R}(\varepsilon)\right] T(\varepsilon) d \varepsilon,
$$

where

$$
T(\varepsilon)=T_{R}^{(1)}+T_{R}^{(2)}=\frac{4 x}{(1+x)^{2}}+\frac{4 C_{1}}{(1+x)^{3} \tilde{\Gamma}} \operatorname{Im} G_{d, d}^{\mathrm{r}}(\varepsilon),
$$

with

$$
\begin{aligned}
C_{1}= & \frac{x^{3}}{1+x}\left(\Gamma_{L} p_{L}^{2}\right)^{2}+x(1-x)\left(\Gamma_{L} p_{L}\right)^{2} \\
& -\Gamma_{L} \Gamma_{R}\left[(1+x)^{3}-x\left(x^{2}+3 x+4\right) p_{L}^{2}\right] .
\end{aligned}
$$

This coincides with Eq. (20) for the current in the twoterminal system with $p_{R}=0$.

\section{APPENDIX D: TIGHT-BINDING MODEL IN FIG. 9}

In the tight-binding model in Fig. 9(a), leads $L$ and $R$ consist of two sites in width and $N$ sites in length $(N \gg 1)$. There are two subbands in the leads, as depicted in Fig. 9(b),

$$
\varepsilon_{ \pm}(q)=\mp t_{1}-2 t \cos q a,
$$

where $t\left(t_{1}\right)$ is the transfer integral in $x(y)$ direction and $a$ is the lattice constant. $q$ is the wave number in the $x$ direction, $q=\pi n /[(N+1) a]$ with $n=1,2, \ldots, N$. The corresponding states are given by Eqs. (43) and (44).

Let us consider the case of two conduction channels in the leads when the Fermi level intersects both the two subbands. They are labeled by $k=(q, \pm)$. In the tunnel Hamiltonian $H_{T}$ in Eq. (4), $V_{L ; q, \pm}=V_{L} \psi_{L ; q, \pm}(-1,1), V_{R ; q, \pm}=$ $V_{R} \psi_{R ; q, \pm}(1,1)$, and $W_{q^{\prime}, \gamma^{\prime} ; q, \gamma}=\sqrt{w_{R ; q^{\prime}, \gamma^{\prime}} w_{L ; q, \gamma}}$, where $w_{L ; q, \gamma}$ and $w_{R: q^{\prime}, \gamma^{\prime}}$ are given by Eqs. (45) and (46), respectively, for $\gamma, \gamma^{\prime}= \pm$. Here, $\psi_{\alpha ; q, \gamma}(j, \ell)=\langle j, \ell \mid \alpha ; q, \gamma\rangle$ is the wave function of the conduction mode $(q, \gamma)$ in lead $\alpha$ : $\psi_{L ; q, \pm}(-1,1)=\psi_{R ; q, \pm}(1,1)=\sin q a / \sqrt{N+1}$ and $\psi_{L ; q, \pm}(-1,2)=\psi_{R ; q, \pm}(1,2)= \pm \sin q a / \sqrt{N+1}$ from Eqs. (43) and (44).

We calculate $\Gamma_{\alpha}, x_{\alpha}$, and $p_{\alpha}$ in Eqs. (6) to (8) at $\varepsilon=E_{\mathrm{F}}$. We focus on lead $L$ because lead $R$ is identical to lead $L$. The density of states for subband \pm is given by

$$
\rho_{ \pm}\left(E_{\mathrm{F}}\right)=\frac{N+1}{\pi} \frac{1}{2 t \sin q_{ \pm} a},
$$

where $q_{ \pm}$is defined by $\varepsilon_{ \pm}\left(q_{ \pm}\right)=E_{\mathrm{F}}$, as depicted in Fig. 9(b),

$$
\begin{aligned}
\Gamma_{L} & =\pi \sum_{\gamma= \pm} \rho_{\gamma}\left(E_{\mathrm{F}}\right)\left(V_{L ; q_{\gamma}, \gamma}\right)^{2} \\
& =\frac{\left(V_{L}\right)^{2}}{2 t}\left(\sin q_{+} a+\sin q_{-} a\right), \\
x_{L} & =\frac{W}{2 t}\left(\sin q_{+} a+\sin q_{-} a\right), \\
\sqrt{\Gamma_{L} x_{L}} p_{L} & =\frac{V_{L} \sqrt{W}}{2 t}\left(\sin q_{+} a-\sin q_{-} a\right),
\end{aligned}
$$

and in consequence we obtain $p_{L}$ in Eq. (47). 
[1] A. Yacoby, M. Heiblum, D. Mahalu, and H. Shtrikman, Coherence and Phase Sensitive Measurements in a Quantum Dot, Phys. Rev. Lett. 74, 4047 (1995).

[2] R. Schuster, E. Buks, M. Heiblum, D. Mahalu, V. Umansky, and H. Shtrikman, Phase measurement in a quantum dot via a double-slit interference experiment, Nature (London) 385, 417 (1997).

[3] O. Entin-Wohlman, A. Aharony, Y. Imry, Y. Levinson, and A. Schiller, Broken Unitarity and Phase Measurements in Aharonov-Bohm Interferometers, Phys. Rev. Lett. 88, 166801 (2002).

[4] A. Aharony, O. Entin-Wohlman, B. I. Halperin, and Y. Imry, Phase measurement in the mesoscopic Aharonov-Bohm interferometer, Phys. Rev. B 66, 115311 (2002).

[5] K. Kobayashi, H. Aikawa, S. Katsumoto, and Y. Iye, Tuning of the Fano Effect Through a Quantum Dot in an Aharonov-Bohm Interferometer, Phys. Rev. Lett. 88, 256806 (2002).

[6] U. Fano, Effects of configuration interaction on intensities and phase shifts, Phys. Rev. 124, 1866 (1961).

[7] A. E. Miroshnichenko, S. Flach, and Y. S. Kivshar, Fano resonances in nanoscale structures, Rev. Mod. Phys. 82, 2257 (2010)

[8] S. Takada, C. Bäuerle, M. Yamamoto, K. Watanabe, S. Hermelin, T. Meunier, A. Alex, A. Weichselbaum, J. von Delft, A. Ludwig, A. D. Wieck, and S. Tarucha, Transmission Phase in the Kondo Regime Revealed in a Two-Path Interferometer, Phys. Rev. Lett. 113, 126601 (2014).

[9] U. Gerland, J. von Delft, T. A. Costi, and Y. Oreg, Transmission Phase Shift of a Quantum Dot with Kondo Correlations, Phys. Rev. Lett. 84, 3710 (2000).

[10] P. G. Silvestrov and Y. Imry, Enhanced Sensitivity of the Transmission Phase of a Quantum Dot to Kondo Correlations, Phys. Rev. Lett. 90, 106602 (2003).

[11] T. Hecht, A. Weichselbaum, Y. Oreg, and J. von Delft, Interplay of mesoscopic and kondo effects for transmission amplitude of few-level quantum dots, Phys. Rev. B 80, 115330 (2009).

[12] Y. Ji, M. Heiblum, D. Sprinzak, D. Mahalu, and H. Shtrikman, Phase evolution in a kondo-correlated system, Science 290, 779 (2000).

[13] Y. Ji, M. Heiblum, and H. Shtrikman, Transmission Phase of a Quantum Dot with Kondo Correlation Near the Unitary Limit, Phys. Rev. Lett. 88, 076601 (2002).

[14] R. Leturcq, D. Sánchez, G. Götz, T. Ihn, K. Ensslin, D. C. Driscoll, and A. C. Gossard, Magnetic Field Symmetry and Phase Rigidity of the Nonlinear Conductance in a Ring, Phys. Rev. Lett. 96, 126801 (2006).

[15] M. Zaffalon, A. Bid, M. Heiblum, D. Mahalu, and V. Umansky, Transmission Phase of a Singly Occupied Quantum Dot in the Kondo Regime, Phys. Rev. Lett. 100, 226601 (2008).

[16] S. Takada, M. Yamamoto, C. Bäuerle, K. Watanabe, A. Ludwig, A. D. Wieck, and S. Tarucha, Measurement of the transmission phase of an electron in a quantum two-path interferometer, Appl. Phys. Lett. 107, 063101 (2015).

[17] S. Takada, M. Yamamoto, C. Bäuerle, A. Alex, J. von Delft, A. Ludwig, A. D. Wieck, and S. Tarucha, Low-temperature behavior of transmission phase shift across a kondo correlated quantum dot, Phys. Rev. B 94, 081303(R) (2016).
[18] B. R. Bułka and P. Stefański, Fano and Kondo Resonance in Electronic Current through Nanodevices, Phys. Rev. Lett. 86, 5128 (2001).

[19] W. Hofstetter, J. König, and H. Schoeller, Kondo Correlations and the Fano Effect in Closed Aharonov-Bohm Interferometers, Phys. Rev. Lett. 87, 156803 (2001).

[20] C. H. Lewenkopf and H. A. Weidenmüller, Kondo temperature for a quantum dot in an Aharonov-Bohm ring, Phys. Rev. B 71, 121309(R) (2005).

[21] R. Yoshii and M. Eto, Scaling analysis for kondo effect in quantum dot embedded in Aharonov-Bohm ring, J. Phys. Soc. Jpn. 77, 123714 (2008).

[22] L. G. G. V. Dias da Silva, N. Sandler, P. Simon, K. Ingersent, and S. E. Ulloa, Tunable Pseudogap Kondo Effect and Quantum Phase Transitions in Aharonov-Bohm Interferometers, Phys. Rev. Lett. 102, 166806 (2009).

[23] J. S. Lim, D. Sánchez, and R. López, Magnetoasymmetric transport in a mesoscopic interferometer: From the weak to the strong coupling regime, Phys. Rev. B 81, 155323 (2010).

[24] T.-F. Fang and H.-G. Luo, Tuning the Kondo and Fano effects in double quantum dots, Phys. Rev. B 81, 113402 (2010).

[25] J. S. Lim, R. López, G. Platero, and P. Simon, Transport properties of a molecule embedded in an Aharonov-Bohm interferometer, Phys. Rev. B 81, 165107 (2010).

[26] J. Malecki and I. Affleck, Influence of interference on the kondo effect in a quantum dot, Phys. Rev. B 82, 165426 (2010).

[27] R. Yoshii and M. Eto, Scaling analysis of kondo screening cloud in a mesoscopic ring with an embedded quantum dot, Phys. Rev. B 83, 165310 (2011).

[28] Y. Komijani, R. Yoshii, and I. Affleck, Interaction effects in Aharonov-Bohm-kondo rings, Phys. Rev. B 88, 245104 (2013).

[29] Z. Shi and Y. Komijani, Conductance of closed and open long Aharonov-Bohm-Kondo rings, Phys. Rev. B 95, 075147 (2017).

[30] Y. Utsumi and K. Saito, Fluctuation theorem in a quantumdot Aharonov-Bohm interferometer, Phys. Rev. B 79, 235311 (2009).

[31] S. Bedkihal and D. Segal, Dynamics of coherences in the interacting double-dot Aharonov-Bohm interferometer: Exact numerical simulations, Phys. Rev. B 85, 155324 (2012).

[32] A. Schuray, L. Weithofer, and P. Recher, Fano resonances in majorana bound states-quantum dot hybrid systems, Phys. Rev. B 96, 085417 (2017).

[33] L. S. Ricco, V. L. Campo, I. A. Shelykh, and A. C. Seridonio, Majorana oscillations modulated by fano interference and degree of nonlocality in a topological superconducting-nanowirequantum-dot system, Phys. Rev. B 98, 075142 (2018).

[34] In Eq. (5), $w_{R, k^{\prime}}$ and $w_{L, k}$ can be replaced by $\lambda w_{R, k^{\prime}}$ and $w_{L, k} / \lambda$ with a constant $\lambda$ for a given $W_{k^{\prime}, k}$. As a result, $x_{L}$ and $x_{R}$ are not well defined by themselves in Eq. (7) in contrast to $p_{L}$ and $p_{R}$ in Eq. (8). $x_{L}$ and $x_{R}$ always appear in the form of $x=x_{L} x_{R}$ in the physical quantities.

[35] T. Kubo, Y. Tokura, T. Hatano, and S. Tarucha, Electron transport through Aharonov-Bohm interferometer with laterally coupled double quantum dots, Phys. Rev. B 74, 205310 (2006).

[36] A. C. Hewson, The Kondo Problem to Heavy Fermions, Cambridge Studies in Magnetism (Cambridge University Press, Cambridge, England, 1993).

[37] A. C. Hewson, Renormalized Perturbation Expansions and Fermi Liquid Theory, Phys. Rev. Lett. 70, 4007 (1993). 
[38] A. C. Hewson, Renormalized perturbation calculations for the single-impurity Anderson model, J. Phys.: Condens. Matter 13, 10011 (2001).

[39] N. Kawakami and A. Okiji, Low temperature thermodynamics of asymmetric anderson model, J. Phys. Soc. Jpn. 52, 1119 (1983).

[40] P. B. Wiegmann and A. M. Tsvelick, Exact solution of the Anderson model: I, J. Phys. C 16, 2281 (1983).

[41] F. D. M. Haldane, Theory of the atomic limit of the Anderson model. i. perturbation expansions re-examined, J. Phys. C 11, 5015 (1978).

[42] A. Ueda, I. Baba, K. Suzuki, and M. Eto, Numerical studies of fano resonance in quantum dots embedded in ab rings, J. Phys. Soc. Jpn., Suppl. A 72, 157 (2003).
[43] Y. Meir and N. S. Wingreen, Landauer Formula for the Current through an Interacting Electron Region, Phys. Rev. Lett. 68, 2512 (1992).

[44] A.-P. Jauho, N. S. Wingreen, and Y. Meir, Time-dependent transport in interacting and noninteracting resonant-tunneling systems, Phys. Rev. B 50, 5528 (1994).

[45] H. Haug and A.-P. Jauho, Quantum Kinetics in Transport and Optics of Semiconductors, 2nd ed., Springer Series in Solid-State Sciences (Springer, Berlin, 2008).

[46] D. C. Langreth, Linear and nonlinear response theory with applications, in Linear and Nonlinear Electron Transport in Solids, edited by J. Devreeese (Springer, New York, 1976), Chap. 1, pp. 3-32. 